\title{
Space-Air-Ground Integrated Network: Outage Performance Analysis
}

\author{
Jia Ye, Student Member, IEEE, Shuping Dang, Member, IEEE, Basem Shihada, Senior Member, IEEE, \\ Mohamed-Slim Alouini, Fellow, IEEE
}

\begin{abstract}
By incorporating the merits of satellite, aerial, and terrestrial communications, the space-air-ground integrated network (SAGIN) emerges in recent years as a promising solution to support seamless, high-rate, and reliable transmission with an extremely larger coverage than a classic terrestrial network. In essence, SAGIN is a cooperative relay network, in which highaltitude platforms (HAPs) and terrestrial base stations (BSs) serve as intermediates relaying signals between end device and satellite. In this paper, we thereby view the SAGIN from the perspective of cooperative communications and introduce relay networking technologies to model and construct the framework of SAGIN. Meanwhile, we take the realistic propagation environment, HAP mobility and mathematical tractability into account and reconstruct the cooperative channel models for SAGIN, including the space-air, space-ground and air-ground links. Based on the constructed framework of SAGIN, we analyze the outage performance and approximate the outage probability as well as asymptotic outage probability in closed form. Numerical results generated by computer simulations verify our analysis and provide insight into the applicability of SAGIN. Although the relaying scenarios considered in this work are simplistic, the good tractability and expandability of the constructed framework provide a solid foundation for further research of advanced systems with complex configurations.
\end{abstract}

Index Terms-Space-air-ground integrated network (SAGIN), cooperative communications, decode-and-forward relaying, outage performance, system modeling.

\section{INTRODUCTION}

A $S$ the concept of the fifth generation (5G) network is getting standardized and mature in recent years, further improvements solely brought by classic terrestrial communications would be limited. As envisioned in [1], there would be five application scenarios supported by the sixth generation (6G) communications: Enhanced Mobile Broadband Plus (eMBB-Plus), Big Communications (BigCom), Secure UltraReliable Low-Latency Communications (SURLLC), ThreeDimensional Integrated Communications (3D-InteCom), and nconventional Data Communications (UCDC). It has been widely acknowledged that without breakthrough, conventional terrestrial wireless communication technologies would be difficult to support these novel applications in $6 \mathrm{G}$ and hardly bring a performance leap from $5 \mathrm{G}$ to $6 \mathrm{G}$ [2]. In light of the limitation of two-dimensional terrestrial communications, the space-airground integrated network (SAGIN) emerges in recent years

J. Ye, S. Dang, B. Shihada, and M.-S. Alouini are with Computer Electrical and Mathematical Science and Engineering Division, King Abdullah University of Science and Technology (KAUST), Thuwal 23955-6900, Kingdom of Saudi Arabia (e-mail: \{jia.ye, shuping.dang, basem.shihada, slim.alouini\}@kaust.edu.sa). as a promising solution to support seamless, high-rate, and reliable transmission with an extremely larger coverage, which raises the network dimension from two to three with an extra dimension of node height [3].

By incorporating the merits of satellite, aerial and terrestrial communications, the SAGIN could play a crucial role in four of the five envisioned application scenarios, i.e., eMBBPlus, BigCom, SURLLC, and 3D-InteCom. A typical SAGIN consists of three layers, which are space, aerial and terrestrial layers. First, a much larger coverage can be provided by satellite communications than that of terrestrial communications at the cost of high latency and low data rate [4], [5]. Second, high-altitude platforms (HAPs) hovering over the aerial layer have the adaptive networking capability, which can adjust the network topology according to the service requirements in real time. On the other hand, the mobility of HAPs could in turn impair the performance of SAGIN without proper coordination and countermeasure [6]. The terrestrial layer supports the classic mobile communications on ground and relies on existing terrestrial infrastructure.

In fact, SAGIN is not a new concept, rather was proposed in 2006 for enhancing the Global Information Grid (GIG) by the Department of Defense, United States [7]. In [7], the rudiments of SAGIN are given, and orbiting satellites and high loiter assets are employed for military communication purposes. However, because near-space and three-dimensional communication technologies were still immature at that time, the predecessor of SAGIN did not attract wide attention. Since 2017, benefiting from the full-fledged unmanned aerial vehicle (UAV) communications, SAGIN architecture gradually becomes a potential solution to support next generation networks. The inherent advantages of SAGIN in terms of coverage, throughput, reliability, and flexibility make it suitable for a number of practical fields in next generation networks, including but not limited to earth observation, intelligent transportation system (ITS), military sensing, emergency communications, rural connectivity [3], [8]-[10].

In [11], software defined SAGIN is employed to cooperate with vehicular networks to support diverse, seamless, efficient, and cost-effective vehicular services. This work marks the resurgence of SAGIN. More general cases incorporating SAGIN and software defined network (SDN) are reported in [12] and [13], which both illustrate the efficiency of the ally. The cross-layer gateway selection problem is discussed in [14]. Bidirectional mission offloading for SAGIN is investigated in [15], which indicates that the satellite, aerial, and terrestrial layers are mutually beneficial. Artificial intelligence (AI) is 
employed to optimize SAGIN for a higher quality of service (QoS) in [16]. Performance analysis of SAGIN using freespace optical (FSO) communications are reported in [17] and [18]. There have also existed two surveys of SAGIN [3], [9].

However, most existing works mentioned above rely on computer simulations to investigate the performance of SAGIN, but hardly provide analytical results. Although some analyses are carried out in [17] and [18], the authors focused on FSO light-of-sight (LoS) links. These FSO LoS links could bring a great challenge for transmitter-receiver alignment and cause severe spatial correlation, which limit the advantageous performance of SAGIN compared to various terrestrial networks. Therefore, to be more realistic and generic, we thereby view the SAGIN from the perspective of cooperative communications and introduce relay networking technologies to model and construct the framework of SAGIN. Also, we take the realistic propagation environment, HAP mobility and mathematical tractability into account and reconstruct the cooperative channel models for SAGIN, including the space-air link, space-ground link and air-ground link. Based on the constructed framework of SAGIN, we analyze the outage performance and approximate the outage probability as well as asymptotic outage probability in closed form. The analytical framework constructed in this work serves as a solid foundation and powerful tool for future research of SAGIN.

The remainder of the paper is organized as follows. In Section II, we detail the system model of the SAGIN considered with several key assumptions and constraints. Then, we carry out outage performance analysis of the SAGIN in Section III by approximating the closed-form expressions of outage probability and deriving their asymptotes. The discussion about the maximum placement distance for HAP is presented in Section IV. Numerical results generated by Monte Carlo simulations are illustrated in Section $\mathrm{V}$ to corroborate our outage performance analysis. Finally, Section VI concludes the paper.

\section{SySTEM MODEL}

\section{A. System Architecture}

We consider a simplistic system model consisting of a geostationary (GEO) satellite, a moving HAP, a terrestrial BS, and a piece of user equipment (UE), which form a threedimensional multi-hop network. We denote the original threedimensional coordinates of the GEO satellite, HAP, terrestrial $\mathrm{BS}$, and UE as $\left(x_{S}, y_{S}, z_{S}\right),\left(x_{H}, y_{H}, z_{H}\right),\left(x_{B}, y_{B}, z_{B}\right)$, and $\left(x_{U}, y_{U}, z_{U}\right)$, respectively. Such a three-dimensional multihop communication scenario is pictorially given in Fig. 1. Assuming all channels are reciprocal, without loss of generality, we only consider the downlink transmission from the GEO satellite to the UE. The transmit power of GEO satellite, HAP, and BS are denoted as $P_{S}, P_{H}$, and $P_{B}$, respectively. All nodes are equipped with a single antenna and operate in a halfduplex mode. For a better interoperability using heterogeneous resources, it is supposed that the GEO satellite, HAP, and BS share the same frequency band ( $\mathrm{L}$ band or S band) and operate over an integrated network in a centralized manner [19]. Due to the mobility of HAP and users, the system adopts

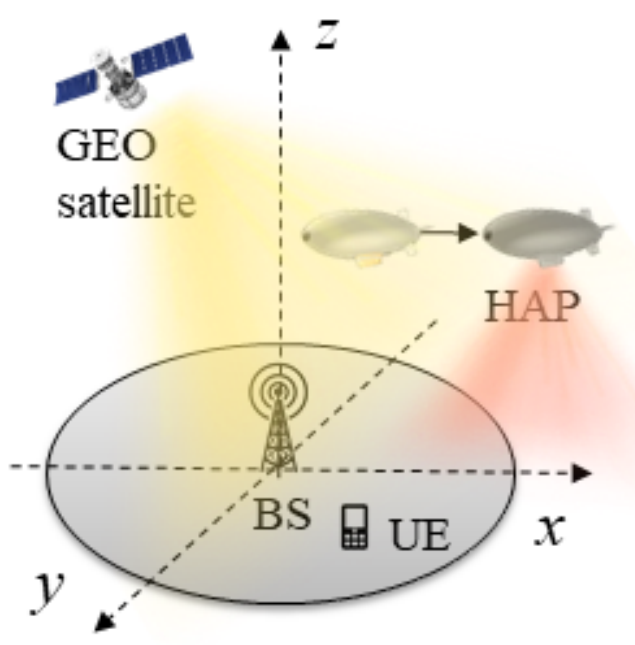

Fig. 1: Three-dimensional multi-hop communication scenario of the considered SAGIN.

the mobile satellite services, which is aim to provide reliable connections to end users that move at high speeds rather than remain stationary [20].

For saving the communication resources, we assume that the UE cannot be directly reached by satellite [21] and the connection must be constructed via HAP or BS that serve as intermediate gateways. Therefore, in the first hop, the GEO satellite broadcasts signal to both BS and HAP. Assuming both BS and HAP adopt the decode-and-forward (DF) relaying protocol, in the second hop, the BS will forward the decoded signal to UE if the received SNR is larger than a threshold $\epsilon_{B}$ or keep silent. The HAP will choose to forward the signal to BS or UE or both depending on its current location and networking mode (details will be given in Section III-A) ${ }^{1}$. In the third hop, the BS will forward the decoded signal after combining the transmission from HAP to the UE if it kept silent in the second hop. Due to the simplicity, selection combining (SC) is adopted for combining signals propagating over multiple paths at the BS and $\mathrm{UE}^{2}$. Perfect time and frequency synchronizations, channel feedback, and CSI acquisition are supposed for the proposed SAGIN system for the analytical simplicity [22]-[26]. Further assuming a well designed transmission protocol implemented at all communication nodes, the interference among multiple transmissions in both time and frequency domains can be circumvented [27], [28].

\section{B. Mobility}

For mobility, both GEO satellite and BS are geostationary, and the movement of UE is assumed to be negligible compared to the scale of SAGIN. However, the mobility of

\footnotetext{
${ }^{1}$ Since the HAP is assumed to act as a DF relay without the need to transmit its own information, the operational delay is negligible compared to the transmission latency over different hops.

${ }^{2}$ Other complex combining techniques, e.g., maximum-ratio combining (MRC), can also be similarly applied and analyzed via the analytical framework constructed in this paper. As the combining technique is not the focus of this work, we omit further in-depth discussion herein.
} 
HAP must be taken into consideration and has an impact on the system performance. In particular, the movement of HAP between the transmissions over the first and second hop yields different transmission distances and thereby different network topologies, which further varies the channel power gain. Therefore, to well model SAGIN, we first need to deal with the mobility of HAP. However, the trajectory of HAP can be regarded to be arbitrary and hard to predict. For generality, we employ the stochastic model to describe the movement behavior of HAP [29]. Before doing so, we first assume the height of the HAP, i.e., $z_{H}$ is a constant, which is justified by the legal requirement that HAP can only operate over a certain layer with a fixed height. Furthermore, constrained by the hardware configuration and energy consumption, the maximum placement of the HAP is regulated to be $r_{H}$. Denoting the new location of the HAP as $\left(x_{H}^{\prime}, y_{H}^{\prime}, z_{H}\right)$, we can mathematically write the constraint as $d_{H}=\sqrt{\left(x_{H}^{\prime}-x_{H}\right)^{2}+\left(y_{H}^{\prime}-y_{H}\right)^{2}+\left(z_{H}-z_{H}\right)^{2}} \leq r_{H}$. Because the height of the HAP is invariant, we can hereby reduce the stochastic model within the aerial layer to twodimensional. According to the network architecture of SAGIN, we can employ the uniform distribution model over the circular region with height $z_{H}$ and radius $r_{H}$ from the center $\left(x_{H}, y_{H}, z_{H}\right)$ to characterize $d_{H}$ and obtain the probability density function (PDF) of $d_{H}$ as [30]

$$
f_{d_{H}}(\lambda)=\left\{\begin{array}{ll}
2 \lambda / r_{H}^{2}, & 0 \leq d_{H} \leq r_{H} \\
0, & \text { otherwise }
\end{array} .\right.
$$

The angle variation of the HAP $\theta_{H}$ is assumed to be uniformly distributed over $[0,2 \pi)$ with the PDF

$$
f_{\theta_{H}}(\lambda)=\left\{\begin{array}{ll}
1 /(2 \pi), & 0 \leq \theta_{H}<2 \pi \\
0, & \text { otherwise }
\end{array} .\right.
$$

As a result, the new coordinate of the HAP can be alternatively written as $\left(x_{H}+d_{H} \cos \left(\theta_{H}\right), y_{H}+d_{H} \sin \left(\theta_{H}\right), z_{H}\right)$. We demonstrate the moving procedure of the HAP with relevant parameters in Fig. 2. For simplicity, we denote the distance between the GEO satellite and HAP and the distance between the GEO satellite and BS when signal is transmitted in the first hop as $d_{S H}=\sqrt{\left(x_{H}-x_{S}\right)^{2}+\left(y_{H}-y_{S}\right)^{2}+\left(z_{H}-z_{S}\right)^{2}}$ and $d_{S B}=\sqrt{\left(x_{B}-x_{S}\right)^{2}+\left(y_{B}-y_{S}\right)^{2}+\left(z_{B}-z_{S}\right)^{2}}$. Similarly, we denote the distance between the HAP and BS and UE when the HAP forwards the signal in the second hop as $d_{H B}^{\prime}=\sqrt{\left(x_{B}-x_{H}^{\prime}\right)^{2}+\left(y_{B}-y_{H}^{\prime}\right)^{2}+\left(z_{B}-z_{H}^{\prime}\right)^{2}}$ and $d_{H U}^{\prime}=\sqrt{\left(x_{U}-x_{H}^{\prime}\right)^{2}+\left(y_{U}-y_{H}^{\prime}\right)^{2}+\left(z_{U}-z_{H}^{\prime}\right)^{2}}$. Because $z_{H} \gg z_{B}, z_{U}$, we assume $z_{B}=z_{U}=0$ for simplicity.

\section{Channel Models}

Due to the heterogeneity of SAGIN, the channels in three layers are diverse and affected by different physical mechanisms. Therefore, it is necessary to discuss them in a separate manner. We classify the channels into three categories: space channel, aerial channel, and terrestrial channel. The space channel refers to the channel between satellite and HAP as well as the channel between satellite and BS. The aerial channel includes the channel between HAP and BS and

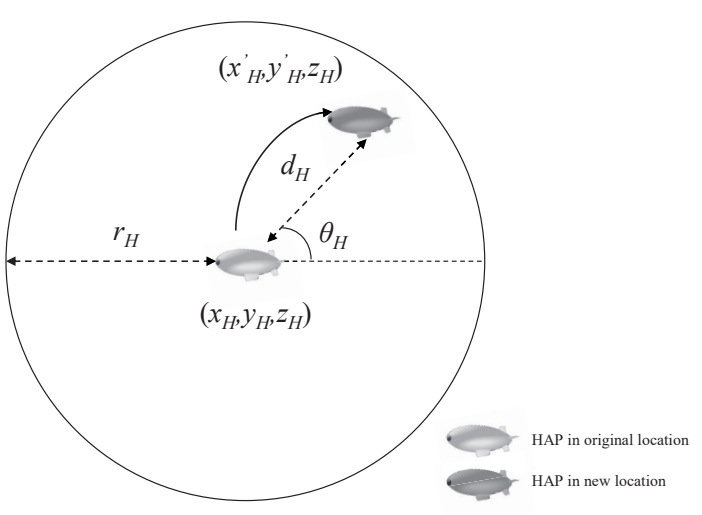

Fig. 2: Movement schematic of the HAP with relevant parameters.

the channel between HAP and UE. Terrestrial channel is the conventional channel between BS and UE, which has been extensively studied in terrestrial communications [31]. Since the movement of HAP is slow when serving users, the Doppler shift is assumed to be well compensated, which has a negligible impact on the SAGIN system [32], [33]. To be general, three propagation attenuation mechanisms are taken into consideration, which are path loss, shadowing and multipath fading.

1) Space Channels: For characterizing space channel, Loo channel model would be the most well-known one with an adequate fit, but the mathematical tractability is relatively poor [34]. In this work, Shadowed-Rician fading model presented in [35] is adopted to describe the satellite signal propagation, which has been proved to be accurate and practical in various frequency bands. Denoting the channel coefficient between satellite and BS as $h_{S B}$, the PDF of the corresponding channel power gain $G_{S B}=\left|h_{S B}\right|^{2}$ is given by [34]

$$
f_{G_{S B}}(\lambda)=\alpha_{S B} \exp \left(-\beta_{S B} \lambda\right){ }_{1} F_{1}\left(m_{S B} ; 1 ; \delta_{S B} \lambda\right),
$$

for $\lambda>0$, where $\alpha_{S B}=\left(\frac{2 b_{S B} m_{S B}}{2 b_{S B} m_{S B}+\Omega_{S B}}\right)^{m_{S B}} /\left(2 b_{S B}\right)$; $\beta_{S B}=\frac{1}{2 b_{S B}}$ and $\delta_{S B}=\frac{\Omega_{S B}}{2 b_{S B}\left(2 b_{S B} m_{S B}+\Omega_{S B}\right)} ; \Omega_{S B}$ and $2 b_{S B}$ are the average power of the LoS and multipath components, respectively; $m_{S B}$ is the fading severity parameter $^{3}$; and ${ }_{1} F_{1}(\cdot ; \cdot ; \cdot)$ is the confluent hypergeometric function of the first kind [36, Eq. (9.14.1)].

Despite the mobility of HAP, the channel model between GEO satellite and HAP is highly similar to the channel model between GEO satellite and $\mathrm{BS}$, given the fact that $z_{S} \gg z_{H}$ [37]. As a consequence of the similarity, we adopt the same channel model as given in (3) for the channel gain pertaining to the space-to-air link $G_{S H}$ by replacing $\alpha_{S B} \beta_{S B}, m_{S B}$, and $\delta_{S B}$ with $\alpha_{S H} \beta_{S H}, m_{S H}$, and $\delta_{S H}$, respectively. As a result of the replacement, the PDF of $G_{S H}$ is given by

$$
f_{G_{S H}}(\lambda)=\alpha_{S H} \exp \left(-\beta_{S H} \lambda\right)_{1} F_{1}\left(m_{S H} ; 1 ; \delta_{S H} \lambda\right) .
$$

\footnotetext{
${ }^{3}$ When $m_{S B}=0$, it represents a complete LoS case; when $0<m_{S B}<$ $\infty$, it stands for the case of both small-scale fading and LoS; when $m_{S B}=\infty$ it denotes the case without LoS.
} 
2) Aerial Channels: For the HAP-to-BS channel, because of the height and elaborate site selection of BS, in general, there exists LoS link between HAP and BS [38]. Therefore, we can assume the channel to be Rician distributed, and the PDF of the channel power gain $G_{H B}$ can be written as [39]

$$
f_{G_{H B}}(\lambda)=\eta^{2} \exp \left(-\left[\eta^{2} \lambda+K\right]\right) I_{0}(2 \eta \sqrt{K \lambda}),
$$

where $\eta=\sqrt{\frac{1+K}{\Omega_{H B}}}$ is the LoS parameter reflecting the strength of the LoS path; $K$ is the Rician factor which corresponds to the ratio of the power of the LoS (specular) component to the average power of the scattered component; $\Omega_{H B}$ is the variance of the signal; $I_{\nu}(\cdot)$ is the modified Bessel function of the first kind with order $\nu$.

For the HAP-to-UE channel, it is supposed that there is no LoS path, because of the complicated electromagnetic propagation environment. Consequently, we adopt the Rayleigh channel model and have the PDF of $G_{H U}$ as

$$
f_{G_{H U}}(\lambda)=\exp (-\lambda)
$$

3) Terrestrial Channel: Finally, for the wireless channel between BS and UE, we again adopt the classic Rayleigh fading channel model with the PDF given by [40]

$$
f_{G_{B U}}(\lambda)=\exp (-\lambda)
$$

\section{Outage Performance Analysis}

\section{A. Outage Scenarios}

There are several scenarios that the UE will experience an outage and cannot receive the signal transmitted by the GEO satellite, depending on the channel qualities and networking modes. We present them all in Fig. 3 and detail them as follows.

1) Outage Scenario I: In this scenario, both of the HAP and BS have well received the broadcast signal from the GEO satellite, which means that the receiving signal-to-noise ratio (SNR) is larger than the HAP and BS outage threshold $\epsilon_{H}$ and $\epsilon_{B}$. Note that, because the BS has already correctly decoded the signal and there is no need to wait for the forwarded signal from the HAP. The BS will directly forward the reencoded signal to the UE in the second transmission phase. Unfortunately, both forwarding links from the HAP and BS to the UE are in outage, which hinders the detection of the forwarded signal at the UE. That is, the receiving SNR at the UE is smaller than the UE outage threshold $\epsilon_{U}{ }^{4}$.

2) Outage Scenario II: In the second scenario, the satelliteto-HAP link in the first hop is in outage, while the transmission over the satellite-to-BS link is successful. However, the forwarded signal in the second hop from the BS to the UE is in outage.

\footnotetext{
${ }^{4}$ Because different devices have different processing capabilities due to various hardware and software configurations, we do not unify the outage thresholds for all receivers at the HAP, BS, and UE for maintaining generality.
}

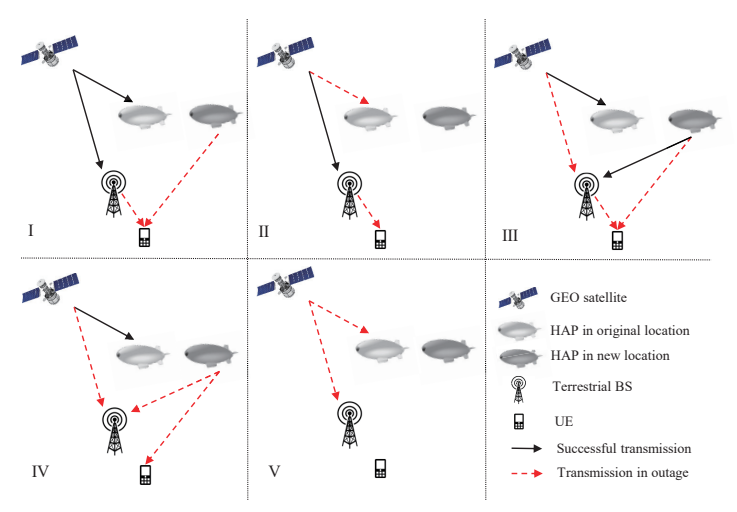

Fig. 3: All scenarios when an outage occurs at the UE.

3) Outage Scenario III: The third scenario refers to the situation that the transmission from the GEO satellite to the BS in the first hop is in outage, but the transmission over the satellite-to-HAP link is successful. Then, in the second hop, the HAP forwards the signal to both the BS and UE. However, the detection of the forwarded signal in the second hop at the $\mathrm{UE}$ is in outage. On the other hand, by $\mathrm{SC}$, the BS is now able to successfully decode the signal forwarded by the HAP and forwards the re-encoded signal to the UE. Unfortunately, outage occurs again over the BS-to-UE link.

4) Outage Scenario $I V$ : In the fourth scenario, the situation in the first hop is exactly the same as the third scenario. However, the forwarded signals in the second hop to the BS and UE are both failed due to outage.

5) Outage Scenario $V$ : The fifth scenario would be the worst one, as both of the transmission over the satellite-toHAP and satellite-to-BS links are in outage in the first hop.

\section{B. Outage Probability Formulation}

By assuming all channel gains are independently distributed, all five outage scenarios are mutually exclusive. To calculate the outage probability, we can first calculate the outage probability corresponding to each scenario. To do so, we define the per-link outage probability for the five links as follows:

$$
\left\{\begin{array}{l}
\Phi_{S H}=\mathbb{P}\left\{P_{S} G_{S H} / d_{S H}^{n_{S H}} N_{0}<\epsilon_{H}\right\} \\
\Phi_{S B}=\mathbb{P}\left\{P_{S} G_{S B} / d_{S B}^{n_{S B}} N_{0}<\epsilon_{B}\right\} \\
\Phi_{H B}=\mathbb{P}\left\{P_{H} G_{H B} /\left(d_{H B}^{\prime}\right)^{n_{H B}} N_{0}<\epsilon_{B}\right\} \\
\Phi_{H U}=\mathbb{P}\left\{P_{H} G_{H U} /\left(d_{H U}^{\prime}\right)^{n_{H U}} N_{0}<\epsilon_{U}\right\} \\
\Phi_{B U}=\mathbb{P}\left\{P_{B} G_{B U} / d_{B U}^{n_{B U}} N_{0}<\epsilon_{U}\right\}
\end{array}\right.
$$

where $\mathbb{P}\{\cdot\}$ denotes the probability of the random event enclosed; $n_{j}, j \in\{S B, S H, H B, H U, B U\}$, denotes path loss exponent for link $j$, which is assumed to be a constant ranging from 2 to 7 depending on the radio propagation environment and terrain; $N_{0}$ is the average power of the complex additive white Gaussian noise (AWGN), which is assumed to follow the same distribution at all receivers. By the definitions of five per-link outage probabilities, we can determine the per-case 
outage probabilities according to the five scenarios illustrated in Fig. 3 as

$$
\left\{\begin{array}{l}
\Psi_{1}=\left(1-\Phi_{S H}\right)\left(1-\Phi_{S B}\right) \Phi_{H U} \Phi_{B U} \\
\Psi_{2}=\Phi_{S H}\left(1-\Phi_{S B}\right) \Phi_{B U} \\
\Psi_{3}=\left(1-\Phi_{S H}\right) \Phi_{S B}\left(1-\Phi_{H B}\right) \Phi_{H U} \Phi_{B U} \\
\Psi_{4}=\left(1-\Phi_{S H}\right) \Phi_{S B} \Phi_{H B} \Phi_{H U} \\
\Psi_{5}=\Phi_{S H} \Phi_{S B}
\end{array} .\right.
$$

By basics of probability theory, we can derive the outage probability of SAGIN as

$$
\Upsilon=\sum_{i=1}^{5} \Psi_{i}
$$

In the following subsection, we derive the approximate expressions in closed form for the five per-link outage probabilities.

\section{Per-Link Outage Probability Derivations}

As analyzed in (9) and (10), we need to first determine the per-link outage probabilities so as to obtain the system outage probability of SAGIN. In this subsection, we hereby presents detailed derivations of per-link outage probabilities $\Phi_{S H}, \Phi_{S B}, \Phi_{H B}, \Phi_{H U}$, and $\Phi_{B U}$ as follows via a set of approximation techniques.

1) GEO-HAP Outage Probability $\Phi_{S H}$ : Based on (4) and (8), $\Phi_{S H}$ can be calculated as

$$
\begin{aligned}
\Phi_{S H}= & \mathbb{P}\left\{G_{S H}<\epsilon_{H} d_{S H}^{n_{S H}} N_{0} / P_{S}\right\} \\
= & \int_{0}^{\epsilon_{H} d_{S H}^{n_{S H}} N_{0} / P_{S}} \\
= & \int_{G_{S H}}(\lambda) \mathrm{d} \lambda \\
& \left.\int_{{ }_{1} F_{1}}{ }^{n_{S H}} N_{S H} / m_{S H} ; 1 ; \delta_{S H} \lambda\right) \mathrm{d} \lambda .
\end{aligned}
$$

For simplicity, we suppose that $m_{S H}$ takes integer values and rewrite the hypergeometric function ${ }_{1} F_{1}\left(m_{S H} ; 1 ; \delta_{S H} \lambda\right)$ through [41, Eq. (41)] as

$$
\begin{aligned}
& { }_{1} F_{1}\left(m_{S H} ; 1 ; \delta_{S H} \lambda\right)=\exp \left(\delta_{S H} \lambda\right) \\
& \times \sum_{k_{S H}=0}^{m_{S H}-1} \frac{(-1)^{k_{S H}}\left(1-m_{S H}\right)_{k_{S H}}\left(\delta_{S H} \lambda\right)^{k_{S H}}}{\left(k_{S H} !\right)^{2}},
\end{aligned}
$$

where $(t)_{k}=t(t+1) \cdots(t+k-1)$ is the Pochhammer symbol [36]. By substituting (12) into (11), the $\Phi_{S H}$ can be calculated as

$$
\begin{aligned}
\Phi_{S H}= & \int_{0}^{\epsilon_{H} d_{S H}^{n_{S H}} N_{0} / P_{S}} \alpha_{S H} \sum_{k_{S H}=0}^{m_{S H}-1} \varsigma\left(k_{S H}\right) \lambda^{k_{S H}} \\
& \times \exp \left[-\left(\beta_{S H}-\delta_{S H}\right) \lambda\right] \mathrm{d} \lambda \\
= & \alpha_{S H} \sum_{k_{S H}=0}^{m_{S H}-1} \varsigma\left(k_{S H}\right)\left(\beta_{S H}-\delta_{S H}\right)^{-k_{S H}-1} \\
& \times \gamma\left(k_{S H}+1, \frac{\left(\beta_{S H} d_{S H}^{n_{S H}}-\delta_{S H} d_{S H}^{n_{S H}}\right) \epsilon_{H} N_{0}}{P_{S}}\right),
\end{aligned}
$$

where $\varsigma\left(k_{S H}\right)=\frac{(-1)^{k_{S H}}\left(1-m_{S H}\right)_{k_{S H}}\left(\delta_{S H}\right)^{k_{S H}}}{\left(k_{S H} !\right)^{2}}$, and $\gamma(\cdot, \cdot)$ is the incomplete gamma function defined in [36, Eq. (8.350.1)].

2) GEO-BS Outage Probability $\Phi_{S B}$ : The calculation of $\Phi_{S B}$ is highly similar to the calculation of $\Phi_{S H}$, which can be directly obtained as

$$
\begin{aligned}
\Phi_{S B}= & \alpha_{S B} \sum_{k_{S B}=0}^{m_{S B}-1} \varsigma\left(k_{S B}\right)\left(\beta_{S B}-\delta_{S B}\right)^{-k_{S B}-1} \\
& \times \gamma\left(k_{S B}+1, \frac{\left(\beta_{S B} d_{S B}^{n_{S B}}-\delta_{S B} d_{S B}^{n_{S B}}\right) \epsilon_{B} N_{0}}{P_{S}}\right),
\end{aligned}
$$

where $\varsigma\left(k_{S B}\right)=\frac{(-1)^{k_{S B}}\left(1-m_{S B}\right)_{k_{S B}}\left(\delta_{S B}\right)^{k_{S B}}}{\left(k_{S B}\right)^{2}}$.

3) HAP-BS Outage Probability $\Phi_{H B}$ : Based on (5), the outage probability conditioned on $d_{H B}^{\prime}$ can be expressed as

$$
\Phi_{H B \mid d_{H B}^{\prime}}=1-Q_{1}\left(\sqrt{2 K}, \eta \sqrt{\frac{2 \epsilon_{B} N_{0}\left(d_{H B}^{\prime}\right)^{n_{H B}}}{P_{H}}}\right)
$$

Considering the mobility of HAP, the transmission distances in the first and second slots are normally different, and the transmission distance in the second slot can be determined as

$$
d_{H B}^{\prime}=\sqrt{\left(x_{H B}+d_{H} \cos \left(\theta_{H}\right)\right)^{2}+\left(y_{H B}+d_{H} \sin \left(\theta_{H}\right)\right)^{2}+z_{H}^{2}},
$$

where $x_{H B}=x_{H}-x_{B}$ and $y_{H B}=y_{H}-y_{B}$. To observe the distribution of $d_{H B}^{\prime}$, we consider the problem as calculating the distribution of the distance from the origin to a point in a circle centered by $\left(x_{H B}, y_{H B}, z_{H}\right)$ with radius $r_{H}$. To demonstrate this problem clearly, we suppose $z_{H}=0$ firstly. Then, this problem is reduced to the $x-y$ plane. It means that we should observe the distance $r$ from the origin to a point randomly distributed in a circle centered by $\left(x_{H B}, y_{H B}\right)$ with radius $r_{H}$, which is shown in Fig. 4. By denoting $r_{B}=\sqrt{x_{H B}^{2}+y_{H B}^{2}}$ and adopting the circlecircle intersection, we calculate the intersection area of HAP distribution regions in (17) on the top of the next page, when $\left|r_{B}-r_{H}\right|<r<r_{B}+r_{H}$.

It should be noted that when $r_{B}<r_{H}, r$ can be smaller than $r_{H}-r_{B}$, which leads to the intersection area shown in Fig. 5. When $0 \leq r<r_{H}-r_{B}$ and $r_{H}>r_{B}$, the another part of intersection area should be the full circle of radius $r$, which can be simplified as

$$
A_{2}(r)=\pi r^{2}
$$

Thus, the CDF of $r$ is calculated by

$$
\begin{aligned}
F_{r}(r)= & \frac{A_{1}(r)}{\pi r_{H}^{2}}, \mathbb{I}\left\{\left|r_{B}-r_{H}\right|<r<r_{B}+r_{H}\right\} \\
& +\frac{A_{2}(r)}{\pi r_{H}^{2}} \mathbb{I}\left\{r<r_{H}-r_{B}, r_{H}>r_{B}\right\},
\end{aligned}
$$

where $\mathbb{I}\{\cdot\}$ is the indicator function. 


$$
\begin{aligned}
A_{1}(r)=r^{2} \arccos \left(\frac{r_{B}^{2}-r_{H}^{2}+r^{2}}{2 r_{B} r}\right) & +r_{H}^{2} \arccos \left(\frac{r_{B}^{2}+r_{H}^{2}-r^{2}}{2 r_{B} r_{H}}\right) \\
& -\frac{1}{2} \sqrt{\left(-r_{B}+r_{H}+r\right)\left(r_{B}-r_{H}+r\right)\left(r_{B}+r_{H}-r\right)\left(r_{B}+r_{H}+r\right)}
\end{aligned}
$$

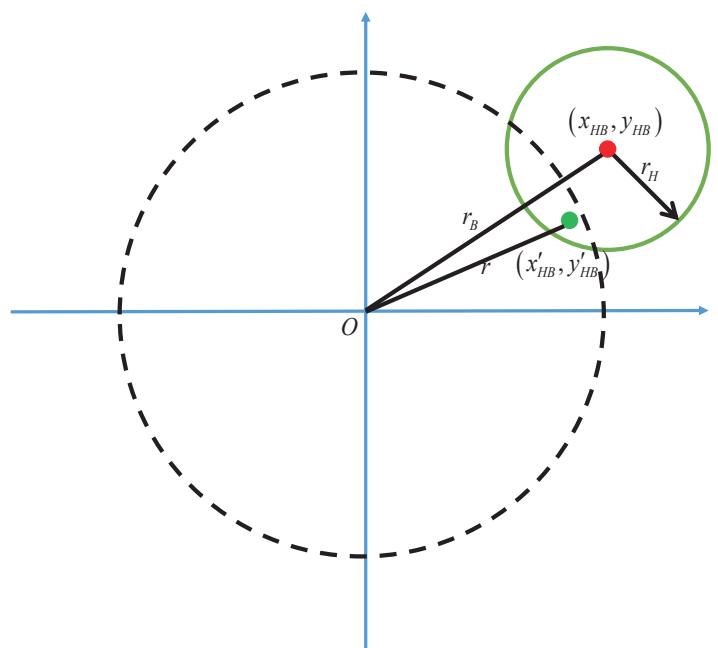

Fig. 4: Distance $r$ from the origin to the HAP when $\left|r_{B}-r_{H}\right|<$ $r<r_{B}+r_{H}$.

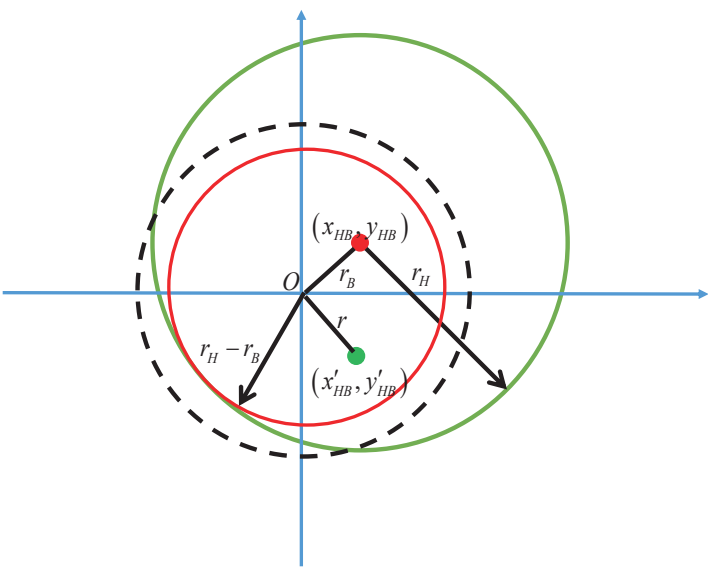

Fig. 5: Distance $r$ from the origin to the HAP when $r<r_{H}-r_{B}$ and $r_{H}>r_{B}$.

Now, taking $z_{H}$ into consideration, we have $d_{H B}^{\prime}=$ $\sqrt{r^{2}+z_{H}^{2}}$. The CDF of $d_{H B}^{\prime}$ can be expressed as

$$
\begin{gathered}
F_{d_{H B}^{\prime}}(x)=\frac{A_{1}\left(\sqrt{x^{2}-z_{H}^{2}}\right)}{\pi r_{H}^{2}} \mathbb{I}\left\{L_{B}<x<U_{B}\right\} \\
+\frac{A_{2}\left(\sqrt{x^{2}-z_{H}^{2}}\right)}{\pi r_{H}^{2}} \mathbb{I}\left\{z_{H}<x<L_{B}, r_{H}>r_{B}\right\},
\end{gathered}
$$

where $L_{B}=\sqrt{\left(r_{B}-r_{H}\right)^{2}+z_{H}^{2}}$ and $\sqrt{\left(r_{B}+r_{H}\right)^{2}+z_{H}^{2}}$.
Furthermore, the PDF of $d_{H B}^{\prime}$ can be further calculated as

$$
\begin{aligned}
& f_{d_{H B}^{\prime}}(x)=\frac{2 x}{r_{H}^{2}} \mathbb{I}\left\{z_{H}<x<L_{B}, r_{H}>r_{B}\right\} \\
& +\frac{2 x}{\pi r_{H}^{2}} \arccos \left(\frac{r_{B}^{2}-r_{H}^{2}-z_{H}^{2}+x^{2}}{2 r_{B} \sqrt{x^{2}-z_{H}^{2}}}\right) \mathbb{I}\left\{L_{B}<x<U_{B}\right\} .
\end{aligned}
$$

Taking the distribution of $d_{H B}^{\prime}$ into consideration, the outage probability of the HAP-BS link can be calculated as

$\Phi_{H B}=1-\int_{\mathcal{R}\left(d_{H B}^{\prime}\right)} Q_{1}\left(\sqrt{2 K}, \eta \sqrt{\lambda_{H B} x^{n_{H B}}}\right) f_{d_{H B}^{\prime}}(x) \mathrm{d} x$,

where $\mathcal{R}\left(d_{H B}^{\prime}\right)$ means the domain of $f_{d_{H B}^{\prime}}(x)$ and $\lambda_{H B}=$ $\frac{2 \epsilon_{B} N_{0}}{P_{S}}$.

To simplify the calculation, we use the approximation for $Q$-function suggested in [42], which is

$$
Q_{1}(a, b) \approx \exp \left(-e^{v(a)} b^{\mu(a)}\right)
$$

where $\mu(a)=2.174-0.592 a+0.593 a^{2}-0.092 a^{3}+0.005 a^{4}$ and $v(a)=-0.840+0.327 a-0.740 a^{2}+0.083 a^{3}-0.004 a^{4}$. Accordingly, we can obtain

$$
Q_{1}\left(\sqrt{2 K}, \eta \sqrt{\lambda_{H B} x^{n_{H B}}}\right) \approx \exp \left(-e^{v} \Theta^{\frac{\mu}{2}} x^{\frac{n_{H B}}{2}}\right),
$$

where $\Theta=\eta^{2} \lambda_{H B}, \mu=2.174-0.937 \sqrt{K}+1.186 K-$ $0.260 K^{\frac{3}{2}}+0.010 K^{2}$ and $v=-0.840+0.809 \sqrt{K}-1.480 K+$ $0.235 K^{\frac{3}{2}}-0.008 K^{2}$.

Thus, $\Phi_{H B}$ can be approximated as

$$
\begin{aligned}
\Phi_{H B}= & \int_{L_{B}}^{U_{B}}\left[1-\exp \left(-e^{v} \Theta^{\frac{\mu}{2}} x^{\frac{n_{H B} \mu}{2}}\right)\right] \frac{2 x}{\pi r_{H}^{2}} \\
& \times \arccos \left(\frac{r_{B}^{2}-r_{H}^{2}-z_{H}^{2}+x^{2}}{2 r_{B} \sqrt{x^{2}-z_{H}^{2}}}\right) \mathrm{d} x \\
& +\int_{z_{H}}^{L_{B}}\left[1-\exp \left(-e^{v} \Theta^{\frac{\mu}{2}} x^{\frac{n_{H B}}{2}}\right)\right] \frac{2 x}{r_{H}^{2}} \mathbb{I}\left\{r_{H}>r_{B}\right\} \mathrm{d} x .
\end{aligned}
$$

In the following derivation, we denote the first integral as $I_{H B}^{1}$, while the second integral as $I_{H B}^{2}$ for simplicity. By taking advantage of (17), $I_{H B}^{1}$ can be approximated as

$$
\begin{aligned}
I_{H B}^{1}= & \frac{A_{1}\left(\sqrt{U_{B}^{2}-z_{H}^{2}}\right)-A_{1}\left(\sqrt{L_{B}^{2}-z_{H}^{2}}\right)}{\pi r_{H}^{2}} \\
& -\int_{L_{B}} \exp \left(-e^{v} \Theta^{\frac{\mu}{2}} x^{\frac{n_{H B}{ }^{\mu}}{2}}\right) \frac{2 x}{\pi r_{H}^{2}} \\
& \times \arccos \left(\frac{r_{B}^{2}-r_{H}^{2}-z_{H}^{2}+x^{2}}{2 r_{B} \sqrt{x^{2}-z_{H}^{2}}}\right) \mathrm{d} x .
\end{aligned}
$$


Since the integral is hard to solve, we use Chebyshev-Gauss quadrature to facilitate further analysis with an adequate accuracy, which is given by $\int_{-1}^{1} \frac{f(x)}{\sqrt{1-x^{2}}} d x \approx \sum_{i=1}^{W_{B}} w_{i} f\left(x_{i}\right)$ with $W_{B}$ sum points, $x_{i}=\cos \left(\frac{2 i-1}{2 W_{B}} \pi\right)$, and weight $w_{i}=\frac{\pi}{W_{B}}$. Thus, $I_{H B}^{1}$ can be rewritten as

$$
\begin{aligned}
& I_{H B}^{1}=\frac{A_{1}\left(\sqrt{U_{B}^{2}-z_{H}^{2}}\right)-A_{1}\left(\sqrt{L_{B}^{2}-z_{H}^{2}}\right)}{\pi r_{H}^{2}} \\
& -\int_{-1}^{1} \frac{2\left(\Gamma_{1} t+\Gamma_{2}\right)}{\pi r_{H}^{2}} \exp \left(-e^{v} \Theta^{\frac{\mu}{2}}\left(\Gamma_{1} t+\Gamma_{2}\right)^{\frac{n_{H B} \mu}{2}}\right) \\
& \times \arccos \left(\frac{r_{B}^{2}-r_{H}^{2}-z_{H}^{2}+\left(\Gamma_{1} t+\Gamma_{2}\right)^{2}}{2 r_{B} \sqrt{\left(\Gamma_{1} t+\Gamma_{2}\right)^{2}-z_{H}^{2}}}\right) \mathrm{d} t \\
& =\frac{A_{1}\left(\sqrt{U_{B}^{2}-z_{H}^{2}}\right)-A_{1}\left(\sqrt{L_{B}^{2}-z_{H}^{2}}\right)}{\pi r_{H}^{2}} \\
& -\Gamma_{1} \sum_{i=1}^{W_{B}} \frac{\pi}{W_{B}} \sqrt{1-\varepsilon_{i}^{2}} \exp \left(-e^{v} \Theta^{\frac{\mu}{2}}\left(\Gamma_{1} \varepsilon_{i}+\Gamma_{2}\right)^{\frac{n_{H B} \mu}{2}}\right) \\
& \times \frac{2\left(\Gamma_{1} \varepsilon_{i}+\Gamma_{2}\right)}{\pi r_{H}^{2}} \arccos \left(\frac{r_{B}^{2}-r_{H}^{2}-z_{H}^{2}+\left(\Gamma_{1} \varepsilon_{i}+\Gamma_{2}\right)^{2}}{2 r_{B} \sqrt{\left(\Gamma_{1} \varepsilon_{i}+\Gamma_{2}\right)^{2}-z_{H}^{2}}}\right) \text {, }
\end{aligned}
$$

where $\Gamma_{1}=\frac{U_{B}-L_{B}}{2} ; \Gamma_{2}=\frac{U_{B}+L_{B}}{2} ; \varepsilon_{i}=$ $\cos \left((2 i-1) \pi / W_{B}\right)$.

Then, $I_{H B}^{2}$ can be calculated as

$$
\begin{aligned}
& I_{H B}^{2}=\int_{z_{H}}^{L_{B}}\left[1-\exp \left(-e^{v} \Theta^{\frac{\mu}{2}} x^{\frac{n_{H B} \mu}{2}}\right)\right] \frac{2 x}{r_{H}^{2}} \mathbb{I}\left\{r_{H}>r_{B}\right\} \mathrm{d} x \\
& =\frac{L_{B}^{2}-z_{H}^{2}}{r_{H}^{2}}-\frac{4}{r_{H}^{2} n_{H B} \mu} e^{-\frac{4 v}{n_{H B}}} \Theta^{-\frac{2}{n_{H B}}} \\
& \times \int_{z_{B}}^{\Psi_{B}} y^{\frac{4}{n_{H B}}-1} \exp (-y) \mathbb{I}\left\{r_{H}>r_{B}\right\} \mathrm{d} y \\
& =\frac{\left(r_{B}-r_{H}\right)^{2}}{r_{H}^{2}}-\frac{4}{r_{H}^{2} n_{H B} \mu} e^{-\frac{4 v}{n_{H B}}} \Theta^{-\frac{2}{n_{H B}}} \\
& \times\left[\Gamma\left(\frac{4}{n_{H B} \mu}, z_{B}\right)-\Gamma\left(\frac{4}{n_{H B} \mu}, \Psi_{B}\right)\right] \mathbb{I}\left\{r_{H}>r_{B}\right\},
\end{aligned}
$$

where $z_{B}=e^{v} \Theta^{\frac{\mu}{2}} z_{H^{2}{ }^{2}}^{n_{H B}}$ and $\Psi_{B}=e^{v} \Theta^{\frac{\mu}{2}} L_{B}^{\frac{n_{H B} \mu}{2}}$.

By substituting (27) and (28) into (25), we can finally approximate $\Phi_{H B}$ in closed form.

4) HAP-UE Outage Probability $\Phi_{H U}$ : Based on (6), the outage probability conditioned on $d_{H U}^{\prime}$ can be expressed as

$$
\Phi_{H U \mid d_{H U}^{\prime}}=1-\exp \left(-\frac{\left(d_{H U}^{\prime}\right)^{n_{H U}} \epsilon_{U} N_{0}}{P_{H}}\right)
$$

Similarly, considering the distribution of $d_{H U}^{\prime}, \Phi_{H U}$ is determined by

$$
\begin{aligned}
\Phi_{H U} & =\int_{\mathcal{R}\left(d_{H U}^{\prime}\right)}\left[1-\exp \left(-\frac{\epsilon_{U} N_{0}}{P_{H}} x^{n_{H U}}\right)\right] f_{d_{H U}^{\prime}}(x) \mathrm{d} x \\
& =\int_{L_{U}}^{U_{U}}\left[1-\exp \left(-\frac{\epsilon_{U} N_{0}}{P_{H}} x^{n_{H U}}\right)\right] \\
& \times \frac{2 x}{\pi r_{H}^{2}} \arccos \left(\frac{r_{U}^{2}-r_{H}^{2}-z_{H}^{2}+x^{2}}{2 r_{U} \sqrt{x^{2}-z_{H}^{2}}}\right) \mathrm{d} x \\
& +\int_{z_{H}}^{L_{U}}\left[1-\exp \left(-\frac{\epsilon_{U} N_{0}}{P_{H}} x^{n_{H U}}\right)\right] \frac{2 x}{r_{H}^{2}} \mathbb{I}\left\{r_{H}>r_{B}\right\} \mathrm{d} x,
\end{aligned}
$$

where $\mathcal{R}\left(d_{H U}^{\prime}\right)$ represents the domain of $f_{d_{H U}^{\prime}}(x)$, and $f_{d_{H U}^{\prime}}(x)$ is obtained by replacing $r_{B}, L_{B}$ and $U_{B}$ in $f_{d_{H U}^{\prime}}(x)$ by $r_{U}=\sqrt{\left(x_{H}-x_{U}\right)^{2}+\left(y_{H}-y_{U}\right)^{2}}, L_{U}=$ $\sqrt{\left(r_{U}-r_{H}\right)^{2}+z_{H}^{2}}$ and $U_{U}=\sqrt{\left(r_{U}+r_{H}\right)^{2}+z_{H}^{2}}$, respectively. Similarly, we denote the first integral and the second integral as $I_{H U}^{1}$ and $I_{H U}^{2}$. And ditto $I_{H U}^{1}$ can be solved through Chebyshev-Gauss quadrature as

$$
\begin{aligned}
I_{H U}^{1}=\frac{A_{1}\left(\sqrt{U_{U}^{2}-z_{H}^{2}}\right)-A_{1}\left(\sqrt{L_{U}^{2}-z_{H}^{2}}\right)}{\pi r_{H}^{2}} \\
-\int_{L_{U}}^{U_{U}} \exp \left(-\frac{\epsilon_{U} N_{0}}{P_{H}} x^{n_{H U}}\right) \frac{2 x}{\pi r_{H}^{2}} \\
\times \arccos \left(\frac{r_{U}^{2}-r_{H}^{2}-z_{H}^{2}+x^{2}}{\left.2 r_{U} \sqrt{x^{2}-z_{H}^{2}}\right) \mathrm{d} x}\right. \\
=\frac{A_{1}\left(\sqrt{U_{U}^{2}-z_{H}^{2}}\right)-A_{1}\left(\sqrt{L_{U}^{2}-z_{H}^{2}}\right)}{\pi r_{H}^{2}} \\
-\Gamma_{3} \sum_{j=1}^{W_{U}} \frac{\pi}{W_{U}} \sqrt{1-\eta_{j}^{2}} \exp \left(-\frac{\epsilon_{U} N_{0}}{P_{H}}\left(\Gamma_{3} \eta_{j}+\Gamma_{4}\right)^{n_{H U}}\right) \\
\times \frac{2\left(\Gamma_{3} \eta_{j}+\Gamma_{4}\right)}{\pi r_{H}^{2}} \arccos \left(\frac{r_{U}^{2}-r_{H}^{2}-z_{H}^{2}+\left(\Gamma_{3} \eta_{j}+\Gamma_{4}\right)^{2}}{2 r_{U} \sqrt{\left(\Gamma_{3} \eta_{j}+\Gamma_{4}\right)^{2}-z_{H}^{2}}}\right),
\end{aligned}
$$

where $\Gamma_{3}=\frac{U_{U}-L_{U}}{2} ; \quad \Gamma_{4}=\frac{U_{U}+L_{U}}{2} ; \eta_{j}=$ $\cos \left((2 j-1) \pi / W_{U}\right)$. 
Similarly, $I_{H U}^{2}$ can be calculated as

$$
\begin{aligned}
I_{H U}^{2}= & \frac{L_{U}^{2}-z_{H}^{2}}{r_{H}^{2}}-\int_{z_{H}}^{L_{U}} \exp \left(-\frac{\epsilon_{U} N_{0}}{P_{H}} x^{n_{H U}}\right) \\
& \times \frac{2 x}{r_{H}^{2}} \mathbb{I}\left\{r_{H}>r_{U}\right\} \mathrm{d} x \\
= & \frac{L_{U}^{2}-z_{H}^{2}}{r_{H}^{2}}-\frac{2}{r_{H}^{2} n_{H B}}\left(\frac{\epsilon_{U} N_{0}}{P_{H}}\right)^{-\frac{2}{n_{H U}}} \\
& \times \int_{z_{U}}^{\Psi_{U}} y^{\frac{2}{n_{H U}}}-1 \exp (-y) \mathbb{I}\left\{r_{H}>r_{U}\right\} \mathrm{d} y \\
= & \frac{L_{U}^{2}-z_{H}^{2}}{r_{H}^{2}}-\frac{2}{r_{H}^{2} n_{H B}}\left(\frac{\epsilon_{U} N_{0}}{P_{H}}\right)^{-\frac{2}{n_{H U}}} \\
& \times\left[\Gamma\left(\frac{2}{n_{H U}}, z_{U}\right)-\Gamma\left(\frac{2}{n_{H U}}, \Psi_{U}\right)\right] \mathbb{I}\left\{r_{H}>r_{U}\right\},
\end{aligned}
$$

where $z_{U}=\frac{\epsilon_{U} N_{0}}{P_{H}} z_{H}^{n_{H U}}$ and $\Psi_{U}=\frac{\epsilon_{U} N_{0}}{P_{H}} L_{U}^{n_{H U}}$.

Finally, substituting (31) and (32) into (30) yields the closed-form approximation for $\Phi_{H U}$.

5) BS-UE Outage Probability $\Phi_{B U}$ : Under the assumption that the channel between BS and UE is the classic Rayleigh, we obtain $\Phi_{B U}$ directly by

$$
\begin{aligned}
\Phi_{B U} & =\mathbb{P}\left\{\frac{P_{B} G_{B U}}{d_{B U}^{n_{B U}} N_{0}}<\epsilon_{U}\right\} \\
& =1-\exp \left(-\frac{d_{B U}^{n_{B U}} \epsilon_{U} N_{0}}{P_{B}}\right) .
\end{aligned}
$$

6) Joint HAP-BS and HAP-UE Outage Probability $\Phi_{H B} \Phi_{H U}$ : It should be noted that we cannot obtain $\Phi_{H B} \Phi_{H U}$ by just multiplying obtained $\Phi_{H B}$ and $\Phi_{H U}$ together. The reason is that $\Phi_{H B}$ and $\Phi_{H U}$ share the same random variables $d_{H}$ and $\theta_{H}$ when considering the mobility of HAP. In other words, the PDFs of $d_{H B}^{\prime}$ and $d_{H U}^{\prime}$ are correlated. By adopting the expressions of the $\Phi_{H B}$ conditioned on $d_{H B}^{\prime}$ and $\Phi_{H U}$ conditioned on $d_{H U}^{\prime}$, we can derive the expression of $\Phi_{H B} \Phi_{H U}$ conditioned on $d_{H B}^{\prime}$ and $d_{H U}^{\prime}$ as

$$
\begin{aligned}
\Phi_{H B \mid d_{H B}^{\prime}} \Phi_{H U \mid d_{H U}^{\prime}} & =\left[1-\exp \left(-e^{v} \Theta^{\frac{\mu}{2}}\left(d_{H B}^{\prime}\right)^{\frac{n_{H B} \mu}{2}}\right)\right] \\
& \times\left[1-\exp \left(-\frac{\epsilon_{U} N_{0}}{P_{H}}\left(d_{H U}^{\prime}\right)^{n_{H U}}\right)\right] .
\end{aligned}
$$

Since $d_{H B}^{\prime}$ and $d_{H U}^{\prime}$ can be re-expressed as

$$
d_{H B}^{\prime}=\sqrt{r_{Z B}+d_{H}^{2}+2 d_{H}\left[y_{H B} \sin \left(\theta_{H}\right)+x_{H B} \cos \left(\theta_{H}\right)\right]},
$$

and

$$
d_{H U}^{\prime}=\sqrt{r_{Z U}+d_{H}^{2}+2 d_{H}\left(y_{H U} \sin \left(\theta_{H}\right)+x_{H U} \cos \left(\theta_{H}\right)\right)},
$$

where $r_{Z B}=r_{B}^{2}+z_{H B}^{2}$ and $r_{Z U}=r_{U}^{2}+z_{H}^{2}$. Therefore, the expression of $\Phi_{H B} \Phi_{H U}$ can be calculated through removing the randomness of $d_{H}$ and $\theta_{H}$, which is given in (37) on the top of next page.

Since the integral is hardly to tackle, we resort to Chebyshev-Gauss quadrature again and obtain the following approximation as

$$
\begin{gathered}
\Phi_{H B} \Phi_{H U}=\sum_{j=1}^{W_{2}} \frac{\pi}{W_{2}} \sqrt{1-\vartheta_{j}^{2}} \sum_{i=1}^{W_{1}} \frac{\pi}{W_{1}} \sqrt{1-\varpi_{i}^{2}} \frac{\vartheta_{j}+1}{4} \\
\times\left[1-\exp \left(-e^{v} \Theta^{\frac{\mu}{2}}\left(r_{Z B}+\Omega_{1}\left(\vartheta_{j}, \varpi_{i}\right)\right)^{\frac{n_{H B} \mu}{4}}\right)\right] \\
\times\left[1-\exp \left(-\frac{\epsilon_{U} N_{0}}{P_{H}}\left(r_{Z U}+\Omega_{2}\left(\vartheta_{j}, \varpi_{i}\right)\right)^{\frac{n_{H U}}{2}}\right)\right],
\end{gathered}
$$

where $\Omega_{1}\left(\vartheta_{j}, \varpi_{i}\right)$ and $\Omega_{2}\left(\vartheta_{j}, \varpi_{i}\right)$ are expressed as

$$
\begin{aligned}
\Omega_{1}\left(\vartheta_{j}, \varpi_{i}\right)= & \left(\frac{r_{H}}{2} \vartheta_{j}+\frac{r_{H}}{2}\right)^{2}-2\left(\frac{r_{H}}{2} \vartheta_{j}+\frac{r_{H}}{2}\right) \\
& \times\left[y_{H B} \sin \left(\pi \varpi_{i}\right)+x_{H B} \cos \left(\pi \varpi_{i}\right)\right],
\end{aligned}
$$

and

$$
\begin{aligned}
\Omega_{2}\left(\vartheta_{j}, \varpi_{i}\right)= & \left(\frac{r_{H}}{2} \vartheta_{j}+\frac{r_{H}}{2}\right)^{2}-2\left(\frac{r_{H}}{2} \vartheta_{j}+\frac{r_{H}}{2}\right) \\
& \times\left[y_{H U} \sin \left(\pi \varpi_{i}\right)+x_{H U} \cos \left(\pi \varpi_{i}\right)\right],
\end{aligned}
$$

where $\varpi_{i}=\cos \left((2 i-1) \pi / W_{1}\right)$ and $\vartheta_{j}=$ $\cos \left((2 j-1) \pi / W_{2}\right)$.

Substituting derived $\Phi_{S H}, \Phi_{S B}, \Phi_{H B}, \Phi_{H U}, \Phi_{B U}$ and $\Phi_{H B} \Phi_{H U}$ into (9), $\Phi_{1}-\Phi_{5}$ can be obtained in closed form. Then, substituting obtained $\Phi_{1}-\Phi_{5}$ into (10), we can finally approximate the outage probability of the SAGIN system in closed form.

\section{Asymptotic Analysis of Outage Performance}

To illustrate the relation among outage performance and a set of system parameters and thereby provide profound insights, we present the asymptotic analysis of outage performance in a per-link manner as follows. In the following, we derive the approximate expressions assuming $\lambda_{S}, \lambda_{H}, \lambda_{B} \rightarrow \infty$. The derived approximate outage probabilities for each link are denoted as $\Phi_{S H}^{A}, \Phi_{S B}^{A}, \Phi_{H B}^{A}, \Phi_{H U}^{A}, \Phi_{B U}^{A}$ respectively.

1) GEO-HAP Outage Probability $\Phi_{S H}^{A}$ : The PDF of $\lambda_{S} G_{S H}$ with $\lambda_{S}=\frac{P_{S}}{N_{0}}$ can be expressed as

$f_{\lambda_{S} G_{S H}}(x)=\frac{\alpha_{S H}}{\lambda_{S}} \exp \left(-\beta_{S H} \frac{x}{\lambda_{S}}\right){ }_{1} F_{1}\left(m_{S H} ; 1 ; \delta_{S H} \frac{x}{\lambda_{S}}\right)$.

As shown in [43], ${ }_{1} F_{1}\left(m_{S H} ; 1 ; \delta_{S H} \frac{x}{\lambda_{S}}\right) \rightarrow 1$ for large $\lambda_{S}$. Thus, the asymptotic PDF of $\lambda_{S} G_{S H}$ can becomes

$$
f_{\lambda_{S} G_{S H}}^{A}(x)=\frac{\alpha_{S H}}{\lambda_{S}} \exp \left(-\beta_{S H} \frac{x}{\lambda_{S}}\right) .
$$

Accordingly, the asymptotic outage probability for GEOHAP $\Phi_{S H}^{A}$ can be calculated as

$$
\begin{aligned}
\Phi_{S H}^{A} & =\int_{0}^{\epsilon_{H} d_{S H}^{n_{S H}}} \frac{\alpha_{S H}}{\lambda_{S}} \exp \left(-\beta_{S H} \frac{x}{\lambda_{S}}\right) \\
& =\frac{\alpha_{S H}}{\beta_{S H}}\left[1-\exp \left(-\beta_{S H} d_{S H}^{n_{S H}} \frac{\epsilon_{H}}{\lambda_{S}}\right)\right] .
\end{aligned}
$$




$$
\begin{aligned}
\Phi_{H B} \Phi_{H U}= & \int_{0}^{r_{H}} \int_{0}^{2 \pi} \frac{d_{H}}{\pi r_{H}^{2}}\left[1-\exp \left(-e^{v} \Theta^{\frac{\mu}{2}}\left[r_{Z B}+d_{H}^{2}+2 d_{H}\left[y_{H B} \sin \left(\theta_{H}\right)+x_{H B} \cos \left(\theta_{H}\right)\right]\right]^{\frac{n_{H B} \mu}{4}}\right)\right] \\
& \times\left[1-\exp \left(-\frac{\epsilon_{U} N_{0}}{P_{H}}\left[r_{Z U}+d_{H}^{2}+2 d_{H}\left[y_{H U} \sin \left(\theta_{H}\right)+x_{H U} \cos \left(\theta_{H}\right)\right]\right]^{\frac{n_{H U}}{2}}\right)\right] \mathrm{d} \theta_{H} \mathrm{~d} d_{H}
\end{aligned}
$$

2) GEO-BS Outage Probability $\Phi_{S B}^{A}$ : Similarly, $\Phi_{S B}^{A}$ can be obtained by replacing $\alpha_{S H}, n_{S H}, \beta_{S H}, \epsilon_{H}$ and $d_{S H}$ by $\alpha_{S B}, n_{S B}, \beta_{S B}, \epsilon_{B}$ and $d_{S B}$ respectively, and we thus have

$$
\Phi_{S B}^{A}=\frac{\alpha_{S B}}{\beta_{S B}}\left[1-\exp \left(-\beta_{S B} d_{S B}^{n_{S B}} \frac{\epsilon_{B}}{\lambda_{S}}\right)\right]
$$

3) HAP-BS Outage Probability $\Phi_{H B}^{A}$ : The PDF of $\lambda_{H} G_{H B}$ with $\lambda_{H}=\frac{P_{H}}{N_{0}}$ can be expressed as

$f_{\lambda_{H} G_{H B}}(x)=\frac{\eta^{2}}{\lambda_{H}} \exp \left(-\eta^{2} \frac{x}{\lambda_{H}}\right) \exp (-K) I_{0}\left(2 \eta \sqrt{K \frac{x}{\lambda_{H}}}\right)$.

By adopting the series representations of the exponential function and the modified Bessel function of the first kind with order 0 , we can have $\exp \left(-\eta^{2} \frac{x}{\lambda_{H}}\right) \approx 1-\eta^{2} \frac{x}{\lambda_{H}}$ and $I_{0}\left(2 \eta \sqrt{K \frac{x}{\lambda_{H}}}\right) \approx 1+\eta^{2} K \frac{x}{\lambda_{H}}$ when $\lambda_{H} \rightarrow \infty$. Thus, the approximated PDF of $\lambda_{H} G_{H B}$ conditioned on $d_{H B}^{\prime}$ can be further simplified as

$f_{\lambda_{H} G_{H B}}^{A}(x)=\frac{\eta^{2}}{\lambda_{H}}\left(1-\eta^{2} \frac{x}{\lambda_{H}}\right) \exp (-K)\left(1+\eta^{2} K \frac{x}{\lambda_{H}}\right)$.

Accordingly, the approximated CDF of $\lambda_{H} G_{H B}$ conditioned on $d_{H B}^{\prime}$ can be obtained as

$$
\begin{aligned}
& F_{\lambda_{H} G_{H B} \mid d_{H B}^{\prime}}^{A}(x)=\int_{0}^{\epsilon_{B}\left(d_{H B}^{\prime}\right)^{n_{H B}}} f_{\lambda_{H} G_{H B}}(x) d x \\
& =\frac{\eta^{2}}{\lambda_{H}} \exp (-K)\left[\left(d_{H B}^{\prime}\right)^{n_{H B}} \epsilon_{B}-\eta^{4} K\left(d_{H B}^{\prime}\right)^{3 n_{H B}} \frac{\epsilon_{B}^{3}}{3 \lambda_{H}^{2}}\right. \\
& \left.\quad+\eta^{2}\left(d_{H B}^{\prime}\right)^{2 n_{H B}}(K-1) \frac{\epsilon_{B}^{2}}{2 \lambda_{H}}\right] .
\end{aligned}
$$

To facilitate the following derivations, we treat $\frac{n_{H B}}{2}=n_{B}$, and $n_{B}$ is an integer. By adopting the expression of $d_{H B}^{\prime}$ shown in (35), $\Phi_{H B}^{A}$ is determined by

$$
\begin{aligned}
\Phi_{H B}^{A}= & \frac{\eta^{2}}{\lambda_{H}} \exp (-K) \int_{0}^{r_{H}} \int_{0}^{2 \pi} \frac{d_{H}}{\pi r_{H}^{2}}\left[\epsilon_{B}\left(d_{H B}^{\prime 2}\right)^{n_{B}}\right. \\
& +\eta^{2}(K-1) \frac{\epsilon_{B}^{2}}{2 \lambda_{H}}\left(d_{H B}^{\prime 2}\right)^{2 n_{B}} \\
& \left.-\eta^{4} K \frac{\epsilon_{B}^{3}}{3 \lambda_{H}^{2}}\left(d_{H B}^{\prime 2}\right)^{3 n_{B}}\right] \mathrm{d} \theta_{H} \mathrm{~d} d_{H} .
\end{aligned}
$$

By adopting the binomial theorem, we can re-express $d_{H B}^{2 n}$ as

$$
\begin{aligned}
d_{H B}^{2 n}= & \left(r_{Z B}+d_{H}^{2}+2 d_{H}\left[y_{H B} \sin \left(\theta_{H}\right)+x_{H B} \cos \left(\theta_{H}\right)\right]\right)^{n} \\
= & \sum_{k=0}^{n}\left(\begin{array}{l}
n \\
k
\end{array}\right) 2^{k} d_{H}^{k}\left(r_{Z B}+d_{H}^{2}\right)^{n-k} \\
& \times\left[y_{H B} \sin \left(\theta_{H}\right)+x_{H B} \cos \left(\theta_{H}\right)\right]^{k} \\
= & \sum_{k=0}^{n}\left(\begin{array}{l}
n \\
k
\end{array}\right) 2^{k} \sum_{i=0}^{n-k}\left(\begin{array}{c}
n-k \\
i
\end{array}\right) r_{Z B}^{n-k-i} d_{H}^{k+2 i} \\
& \times\left[y_{H B} \sin \left(\theta_{H}\right)+x_{H B} \cos \left(\theta_{H}\right)\right]^{k}
\end{aligned}
$$

Finally, $\Phi_{H B}^{A}$ can be derived as

$$
\begin{aligned}
\Phi_{H B}^{A} & =\frac{\eta^{2}}{\lambda_{H}} \exp (-K)\left[\epsilon_{B} \mathcal{F}_{1}\left(r_{Z B}, x_{H B}, y_{H B}, n_{B}\right)\right. \\
& -\eta^{4} K \frac{\epsilon_{B}^{3}}{3 \lambda_{H}^{2}} \mathcal{F}_{1}\left(r_{Z B}, x_{H B}, y_{H B}, 3 n_{B}\right) \\
& \left.+\eta^{2}(K-1) \frac{\epsilon_{B}^{2}}{2 \lambda_{H}} \mathcal{F}_{1}\left(r_{Z B}, x_{H B}, y_{H B}, 2 n_{B}\right)\right],
\end{aligned}
$$

where $\mathcal{F}_{1}(\cdot, \cdot, \cdot, \cdot)$ is given by

$$
\begin{aligned}
& \mathcal{F}_{1}(a, b, c, n)=\mathcal{S}_{F} \\
& \times \int_{0}^{r_{H}} \int_{0}^{2 \pi} d_{H}^{k+2 i+1}\left[c \sin \left(\theta_{H}\right)+b \cos \left(\theta_{H}\right)\right]^{k} \mathrm{~d} \theta_{H} \mathrm{~d} d_{H} \\
& =\mathcal{S}_{F} \frac{r_{H}^{k+2 i+2}}{k+2 i+2} \int_{0}^{2 \pi}\left[c \sin \left(\theta_{H}\right)+b \cos \left(\theta_{H}\right)\right]^{k} \mathrm{~d} \theta_{H} \\
& =\mathcal{S}_{F} \frac{r_{H}^{k+2 i+2}}{k+2 i+2} \begin{cases}\frac{(k-1) ! !}{(k) ! !} \cdot 2 \pi\left(b^{2}+c^{2}\right)^{\frac{k}{2}}, k \text { is even } \\
0, & k \text { is odd }\end{cases}
\end{aligned}
$$

where $\mathcal{S}_{F}=\frac{1}{\pi r_{H}^{2}} \sum_{k=0}^{n}\left(\begin{array}{l}n \\ k\end{array}\right) 2^{k} \sum_{i=0}^{n-k}\left(\begin{array}{c}n-k \\ i\end{array}\right) a^{n-k-i}$ and (.)!! represents double factorial notation.

4) HAP-UE Outage Probability $\Phi_{H U}^{A}$ : Considering $\lambda_{H} \rightarrow$ $\infty$, the exponential $\exp \left(-\frac{x}{\lambda_{H}}\right) \approx 1-\frac{x}{\lambda_{H}}$. Thus, the approximated PDF of $\lambda_{H} G_{H U}$ can be reduced as

$$
f_{\lambda_{H} G_{H U}}^{A}(x)=\frac{1}{\lambda_{H}}\left(1-\frac{x}{\lambda_{H}}\right) \text {. }
$$

Accordingly, the approximated $\mathrm{CDF}$ of $\lambda_{H} G_{H U}$ conditioned on $d_{H U}^{\prime}$ can be calculated as

$$
\begin{aligned}
F_{\lambda_{H} G_{H U} \mid d_{H U}^{\prime}}^{A}(x) & =\int_{0}^{\epsilon_{U}\left(d_{H U}^{\prime}\right)^{n_{H U}}} f_{\lambda_{H} G_{H U}}^{A}(x) \mathrm{d} x \\
& =\frac{1}{\lambda_{H}}\left(\left(d_{H U}^{\prime}\right)^{n_{H U}} \epsilon_{U}-\left(d_{H U}^{\prime}\right)^{2 n_{H U}} \frac{\epsilon_{U}^{2}}{2 \lambda_{H}}\right) .
\end{aligned}
$$


By assuming $n_{U}=\frac{n_{H U}}{2}$ to be an integer and using the expression of $d_{H U}^{\prime}$ shown in (36), we derive $\Phi_{H U}^{A}$ as

$$
\begin{aligned}
\Phi_{H U}^{A}= & \frac{1}{\lambda_{H}} \int_{0}^{r_{H}} \int_{0}^{2 \pi} \frac{d_{H}}{\pi r_{H}^{2}}\left[\epsilon_{U}\left(d_{H U}^{\prime 2}\right)^{n_{U}}\right. \\
& \left.-\frac{\epsilon_{U}^{2}}{2 \lambda_{H}}\left(d_{H U}^{\prime 2}\right)^{2 n_{U}}\right] \mathrm{d} \theta_{H} \mathrm{~d} d_{H} .
\end{aligned}
$$

Using defined function $\mathcal{F}_{1}(\cdot, \cdot, \cdot, \cdot)$, we obtain the asymptotic outage probability $\Phi_{H U}^{A}$ by

$$
\begin{aligned}
\Phi_{H U}^{A}= & \frac{1}{\lambda_{H}}\left[\epsilon_{U} \mathcal{F}_{1}\left(r_{Z U}, x_{H U}, y_{H U}, n_{U}\right)\right. \\
& \left.-\frac{\epsilon_{U}^{2}}{2 \lambda_{H}} \mathcal{F}_{1}\left(r_{Z U}, x_{H U}, y_{H U}, 2 n_{U}\right)\right] .
\end{aligned}
$$

5) BS-UE Outage Probability $\Phi_{B U}^{A}$ : The asymptotic expression of $\Phi_{B U}^{A}$ is given by

$$
\Phi_{B U}^{A}=\frac{1}{\lambda_{H}}\left(\left(d_{B U}\right)^{n_{B U}} \epsilon_{U}-\left(d_{B U}\right)^{2 n_{B U}} \frac{\epsilon_{U}^{2}}{2 \lambda_{H}}\right) .
$$

By substituting (43), (44), (50), (55) and (56) into (9) and then into (10), the overall asymptotic outage probability of the whole system can be obtained.

6) Joint HAP-BS and HAP-UE Outage Probability $\Phi_{H B}^{A} \Phi_{H U}^{A}$ : Similarly, we re-calculate the asymptotic expression of $\Phi_{H B}^{A} \Phi_{H U}^{A}$ considering the influence of $d_{H}$ and $\theta_{H}$ on $d_{H B}^{\prime}$ and $d_{H U}^{\prime}$ jointly. By taking advantage of (47) and (53), $\Phi_{H B}^{A} \Phi_{H U}^{A}$ can be derived in (57) on the top of next page, where $\sigma_{1}=\epsilon_{B} \epsilon_{U} ; \sigma_{2}=\frac{\epsilon_{B} \epsilon_{U}^{2}}{2 \lambda_{H}} ; \sigma_{3}=\frac{\eta^{4} K \epsilon_{U} \epsilon_{B}^{3}}{3 \lambda_{H}^{2}}$; $\sigma_{4}=\frac{\eta^{4} K \epsilon_{U}^{2} \epsilon_{B}^{3}}{6 \lambda_{H}^{3}} ; \sigma_{5}=\frac{\eta^{2}(K-1) \epsilon_{U} \epsilon_{B}^{2}}{2 \lambda_{H}} ; \sigma_{6}=\frac{\eta^{2}(K-1) \epsilon_{U}^{2} \epsilon_{B}^{2}}{4 \lambda_{H}^{2}} ;$ $\psi=\frac{\eta^{2}}{\pi r_{H}^{2} \lambda_{H}^{2}} \exp (-K)$ and $\mathcal{F}_{2}(\cdot, \cdot)$ is given by

$$
\mathcal{F}_{2}\left(n_{1}, n_{2}\right)=\mathcal{S}_{B U} \frac{r_{H}^{k+2 i+1+j+2 m}}{k+2 i+2+j+2 m} \mathcal{F}_{3}(k, j),
$$

where $\mathcal{S}_{B U}=\frac{1}{\pi r_{H}^{2}} \sum_{k=0}^{n_{1}}\left(\begin{array}{c}n_{1} \\ k\end{array}\right) 2^{k} \sum_{i=0}^{n_{1}-k}\left(\begin{array}{c}n_{1}-k \\ i\end{array}\right) r_{Z B}^{n_{1}-k-i}$

$\sum_{j=0}^{n_{2}}\left(\begin{array}{c}n_{2} \\ j\end{array}\right) 2^{j} \sum_{m=0}^{n_{2}-j}\left(\begin{array}{c}n_{2}-j \\ m\end{array}\right) r_{Z U}^{n_{2}-j-m}$ and $\mathcal{F}_{3}(\cdot, \cdot)$ is given as

$\mathcal{F}_{3}(a, b)=\mathcal{S}_{T} \int_{0}^{2 \pi} \sin ^{k+i}\left(\theta_{H}\right) \cos ^{a+b-k-i}\left(\theta_{H}\right) \mathrm{d} \theta_{H}$

$=\mathcal{S}_{T} \begin{cases}\mathcal{F}_{3,1}(k+i, a+b-k-i), & a+b-k-i \text { is even } \\ 0, & a+b-k-i \text { is odd }\end{cases}$

where $\mathcal{S}_{T}=\sum_{k=0}^{a}\left(\begin{array}{l}a \\ k\end{array}\right) \sum_{i=0}^{b}\left(\begin{array}{l}b \\ i\end{array}\right) y_{H B}^{k} y_{H U}^{i} x_{H B}^{a-k} x_{H U}^{b-i}$, and $\mathcal{F}_{3,1}(\cdot, \cdot)$ is defined as

$$
\begin{aligned}
& \mathcal{F}_{3,1}(a, b)=\frac{(b-1) ! !}{(b+a)(b+a-2) \ldots(a+2)} \int_{0}^{2 \pi} \sin ^{a}(x) \mathrm{d} x \\
& =\frac{(b-1) ! !}{(b+a)(b+a-2) \ldots(a+2)} \begin{cases}\frac{\pi(a-1) ! !}{2^{\frac{a}{2}-1}\left(\frac{a}{2}\right) !}, & a \text { is even } \\
0, & a \text { is odd }\end{cases}
\end{aligned}
$$

\section{E. Analysis for More General Channel}

In practice, the fading channel models among HAP, BS and user are mainly dependent on the distance and propagation environment. In general, they can be all modeled by the Rician distribution with the Rician shape parameter $K_{j}\left(d_{j}\right)$, $j \in\{H B, H U, B U\}$. The Rician shape parameter $K_{j}\left(d_{j}\right)$ is defined as the ratio of the power of the LoS (specular) component to the average power of the scattered components. The Rician shape parameter $K_{j}\left(d_{j}\right)$ is related to the distance $d_{j}$ and propagation environment. For simplicity, we treat $K_{j}\left(d_{j}\right)$ as a function of $d_{j}$ in the following analysis. The case $K_{j}\left(d_{j}\right)=0$ corresponds to the most severe fading, and in this limiting case, the gain magnitude is said to be Rayleigh distributed. Under these assumptions, the PDF of the channel gain of channel $j, j \in\{H B, H U, B U\}$ can be re-expressed as

$$
f_{G_{j}}(\lambda)=\eta_{j}^{2} \exp \left(-\left[\eta_{j}^{2} \lambda+K_{j}\left(d_{j}\right)\right]\right) I_{0}\left(2 \eta \sqrt{K_{j}\left(d_{j}\right) \lambda}\right),
$$

where $\eta_{j}=\sqrt{\frac{1+K_{j}\left(d_{j}\right)}{\Omega_{H B}}}$ is the LoS parameter reflecting the strength of the LoS path; $\Omega_{j}$ is the variance of the signal for channel $j$.

1) HAP-BS Outage Probability $\Phi_{H B}^{G}$ : Considering the distribution of $d_{H B}^{\prime}$, the outage probability between HAP and BS can be expressed as

$\Phi_{H B}^{G}=1-$

$\int_{\mathcal{R}(x)} Q_{1}\left(\sqrt{2 K_{H B}(x)}, \eta_{H B}(x) \sqrt{\lambda_{H B} x^{n_{H B}}}\right) f_{d_{H B}^{\prime}}(x) \mathrm{d} x$,

where $\mathcal{R}\left(d_{H B}^{\prime}\right)$ means the domain of $f_{d_{H B}^{\prime}}(x)$ and $\eta_{H B}(x)=$ $\sqrt{\frac{1+K_{H B}(x)}{\Omega_{H B}}}$.

2) HAP-UE Outage Probability $\Phi_{H U}^{G}$ : Similarly, considering the distribution of $d_{H U}^{\prime}$, the outage probability between HAP and UE can be expressed as

$\Phi_{H U}^{G}=1-$

$\int_{\mathcal{R}(x)} Q_{1}\left(\sqrt{2 K_{H U}(x)}, \eta_{H U}(x) \sqrt{\lambda_{H U} x^{n_{H U}}}\right) f_{d_{H U}^{\prime}}(x) \mathrm{d} x$,

where $\mathcal{R}\left(d_{H U}^{\prime}\right)$ means the domain of $f_{d_{H U}^{\prime}}(x)$ and $\eta_{H U}(x)=$ $\sqrt{\frac{1+K_{H U}(x)}{\Omega_{H U}}}$.

3) BS-UE Outage Probability $\Phi_{B U}^{G}$ : Since the $d_{B U}$ is assumed to be fixed in the SAGIN model, $\Phi_{B U}^{G}$ can be obtained as

$\Phi_{B U}^{G}=1-Q_{1}\left(\sqrt{2 K_{B U}\left(d_{B U}\right)}, \eta_{B U}\left(d_{B U}\right) \sqrt{\lambda_{B U} x^{n_{B U}}}\right)$,

where $\eta_{B U}(x)=\sqrt{\frac{1+K_{B U}\left(d_{B U}\right)}{\Omega_{B U}}}$.

4) Joint HAP-BS and HAP-UE Outage Probability $\Phi_{H B}^{G} \Phi_{H U}^{G}$ : By adopting the expressions of $\Phi_{H B}$ conditioned on $d_{H B}^{\prime}\left(d_{H}, \theta_{H}\right)$ and $\Phi_{H U}$ conditioned on $d_{H U}^{\prime}\left(d_{H}, \theta_{H}\right)$, and removing the randomness of $d_{H B}^{\prime}\left(d_{H}, \theta_{H}\right)$ and $d_{H U}^{\prime}\left(d_{H}, \theta_{H}\right), \Phi_{H B}^{G} \Phi_{H U}^{G}$ can be determined in (65) on the top of the next page. 


$$
\begin{aligned}
& \Phi_{H B}^{A} \Phi_{H U}^{A}=\frac{\eta^{2}}{\pi r_{H}^{2} \lambda_{H}^{2}} \exp (-K) \int_{0}^{r_{H}} \int_{0}^{2 \pi} d_{H}\left[\sigma_{1}\left(d_{H B}^{\prime 2}\right)^{n_{B}}\left(d_{H U}^{\prime 2}\right)^{n_{U}}-\sigma_{2}\left(d_{H B}^{\prime 2}\right)^{n_{B}}\left(d_{H U}^{\prime 2}\right)^{2 n_{U}}\right. \\
& \left.-\sigma_{3}\left(d_{H B}^{\prime 2}\right)^{3 n_{B}}\left(d_{H U}^{\prime 2}\right)^{n_{U}}+\sigma_{4}\left(d_{H B}^{\prime 2}\right)^{3 n_{B}}\left(d_{H U}^{\prime 2}\right)^{2 n_{U}}+\sigma_{5}\left(d_{H B}^{\prime 2}\right)^{2 n_{B}}\left(d_{H U}^{\prime 2}\right)^{n_{U}}-\sigma_{6}\left(d_{H B}^{\prime 2}\right)^{2 n_{B}}\left(d_{H U}^{\prime 2}\right)^{2 n_{U}}\right] \mathrm{d} \theta_{H} \mathrm{~d} d_{H} \\
& =\psi\left[\sigma_{1} \mathcal{F}_{2}\left(n_{B}, n_{U}\right)-\sigma_{2} \mathcal{F}_{2}\left(n_{B}, 2 n_{U}\right)-\sigma_{3} \mathcal{F}_{2}\left(3 n_{B}, n_{U}\right)+\sigma_{4} \mathcal{F}_{2}\left(3 n_{B}, 2 n_{U}\right)+\sigma_{5} \mathcal{F}_{2}\left(2 n_{B}, n_{U}\right)-\sigma_{6} \mathcal{F}_{2}\left(2 n_{B}, 2 n_{U}\right)\right]
\end{aligned}
$$

$$
\begin{aligned}
\Phi_{H B}^{G} \Phi_{H U}^{G} & =\int_{0}^{R} \int_{0}^{2 \pi} \frac{d_{H}}{\pi r_{H}^{2}}\left[1-Q_{1}\left(\sqrt{2 K_{H B}\left(d_{H B}^{\prime}\left(d_{H}, \theta_{H}\right)\right)}, \eta_{H B}\left(d_{H B}^{\prime}\left(d_{H}, \theta_{H}\right)\right) \sqrt{\lambda_{H B} d_{H B}^{\prime}\left(d_{H}, \theta_{H}\right)^{n_{H B}}}\right)\right] \\
& \times\left[1-Q_{1}\left(\sqrt{2 K_{H U}\left(d_{H U}^{\prime}\left(d_{H}, \theta_{H}\right)\right)}, \eta_{H U}\left(d_{H U}^{\prime}\left(d_{H}, \theta_{H}\right)\right) \sqrt{\lambda_{H U} d_{H U}^{\prime}\left(d_{H}, \theta_{H}\right)^{n_{H U}}}\right)\right] \mathrm{d} \theta_{H} \mathrm{~d} d_{H} \cdot
\end{aligned}
$$

\section{Optimization of the Spatial Distribution of HAP}

As shown in the previous analysis, the HAP, acting as an intermediate node with mobility, bridges the satellite communications and terrestrial communications and is of key importance. It is thereby worth investigating the impact of its mobility on the system outage performance of SAGIN. In this section, we study the optimization problem of the spatial distribution of HAP.

The optimization problem can be formulated as

$$
\begin{aligned}
\text { Find } & : r_{H} \\
\text { Minimization }: & \Upsilon \\
\text { Subject to }: & r_{H} \geq 0 .
\end{aligned}
$$

It can be easily found that $r_{H}$ only has an influence on $\Phi_{H B}$ and $\Phi_{H U}$. Thus, the objective function is reduced to $O_{\text {new }}=$ $\Psi_{1}+\Psi_{3}+\Psi_{4}$, which can be re-expressed as

$$
\begin{aligned}
O_{\text {new }}= & \Psi_{1}+\Psi_{3}+\Psi_{4} \\
= & \left(1-\Phi_{S H}\right)\left(1-\Phi_{S B}\right) \Phi_{H U} \Phi_{B U} \\
& +\left(1-\Phi_{S H}\right) \Phi_{S B}\left(1-\Phi_{H B}\right) \Phi_{H U} \Phi_{B U} \\
& +\left(1-\Phi_{S H}\right) \Phi_{S B} \Phi_{H B} \Phi_{H U} \\
= & \Phi_{1} \Phi_{H U}+\Phi_{2} \Phi_{H B} \Phi_{H U},
\end{aligned}
$$

where $\Phi_{1}=\left(1-\Phi_{S H}\right) \Phi_{B U}$ and $\Phi_{2}=(1-$ $\left.\Phi_{S H}\right) \Phi_{S B}\left(1-\Phi_{B U}\right)$. Thus, the optimization problem becomes

Find : $r_{H}$

$$
\begin{aligned}
& \text { Minimization : } \Phi_{1} \Phi_{H U}+\Phi_{2} \Phi_{H B} \Phi_{H U}, \\
& \text { Subject to : } r_{H} \geq 0 .
\end{aligned}
$$

By observing the objective function, we should find $r_{H}$ to minimize $\Phi_{H B}$ and $\Phi_{H U}$. Since the obtained expressions for $\Phi_{H B}$ and $\Phi_{H U}$ are mathematically intractable, we must find another alternative objective functions to approximate $\Phi_{H B}$ and $\Phi_{H U}$ and provide a suboptimal solution instead. Based on the definition shown in (15) and (29), $\Phi_{H B}$ and $\Phi_{H U}$ can be re-expressed as

$$
\Phi_{H B}=1-\mathbb{E}_{d_{H B}^{\prime}}\left\{\exp \left(-e^{v} \Theta^{\frac{\mu}{2}}\left(d_{H U}^{\prime 2}\right)^{\frac{n_{H B} \mu}{4}}\right)\right\},
$$

and

$$
\Phi_{H U}=1-\mathbb{E}_{d_{H U}^{\prime}}\left\{\exp \left(-\frac{\left(d_{H U}^{\prime 2}\right)^{n_{H U}} \epsilon_{U} N_{0}}{P_{H}}\right)\right\},
$$

where $\mathbb{E}_{x_{1}, x_{2}, \ldots}\{\cdot\}$ denotes the mean of the enclosed expression averaged over random variables $x_{1}, x_{2}, \ldots$, and we let $\mathbb{E}\{\cdot\}=\mathbb{E}_{x}\{x\}$ for simplicity. Then, we formulate an alternative optimization problem to approximate the original optimization problem by replacing all instantaneous $d_{H B}^{\prime}$ and $d_{H U}^{\prime}$ by their averages as follows:

$$
\begin{aligned}
\mathbb{E}\left\{d_{H B}^{\prime 2}\right\}= & r_{Z B}+\mathbb{E}_{d_{H}}\left\{d_{H}^{2}\right\}+2 y_{H B} \mathbb{E}_{d_{H}, \theta_{H}}\left\{d_{H} \sin \left(\theta_{H}\right)\right\} \\
& +2 x_{H B} \mathbb{E}_{d_{H}, \theta_{H}}\left\{d_{H} \cos \left(\theta_{H}\right)\right\} \\
= & r_{Z B}+\frac{r_{H}^{2}}{2}
\end{aligned}
$$

and

$$
\mathbb{E}\left\{d_{H U}^{\prime 2}\right\}=r_{Z U}+\frac{r_{H}^{2}}{2} .
$$

Thus, we obtain the substitutions of $\Phi_{H B}$ and $\Phi_{H U}$ as

$$
\Phi_{H B}^{n e w}=1-\exp \left(-e^{v} \Theta^{\frac{\mu}{2}}\left(r_{Z B}+\frac{r_{H}^{2}}{2}\right)^{\frac{n_{H B} \mu}{4}}\right),
$$

and

$$
\Phi_{H U}^{n e w}=1-\exp \left(-\frac{\left(r_{Z U}+\frac{r_{H}^{2}}{2}\right)^{n_{H U}} \epsilon_{U} N_{0}}{P_{H}}\right) .
$$

It is obvious that $\Phi_{H B}^{n e w}$ and $\Phi_{H U}^{n e w}$ increase as $r_{H}$ increases. Thus, the new objective function formulated by $\Phi_{1} \Phi_{H U}^{n e w}+$ $\Phi_{2} \Phi_{H B}^{n e w} \Phi_{H U}^{n e w}$ would also decrease as $r_{H}$ decreases. Consequently, the suboptimal solution of $r_{H}$ should be as small as possible, which will be verified in Section V.

\section{NumericAl RESUlts}

In this section, we present the numerical results produced by Monte Carlo simulations to verify the correctness of our analysis. Following the parameters adopted in [44]-[46], the main parameters in our simulations are set as: $b_{S B}=b_{S H}=\Omega_{S B}=$ $\Omega_{S H}=15 \mathrm{~dB}, m_{S B}=m_{S H}=2, \Omega_{H B}=5 \mathrm{~dB}, K=-10$ $\mathrm{dB}, n_{S B}=n_{S H}=n_{H B}=n_{H U}=n_{B U}=2, N_{0}=-94$ $\mathrm{dBW}, \epsilon_{B}=\epsilon_{H}=\epsilon_{U}=1 \mathrm{~dB}, r_{H}=2000 \mathrm{~m},\left(x_{S}, y_{S}, z_{S}\right)=$ 


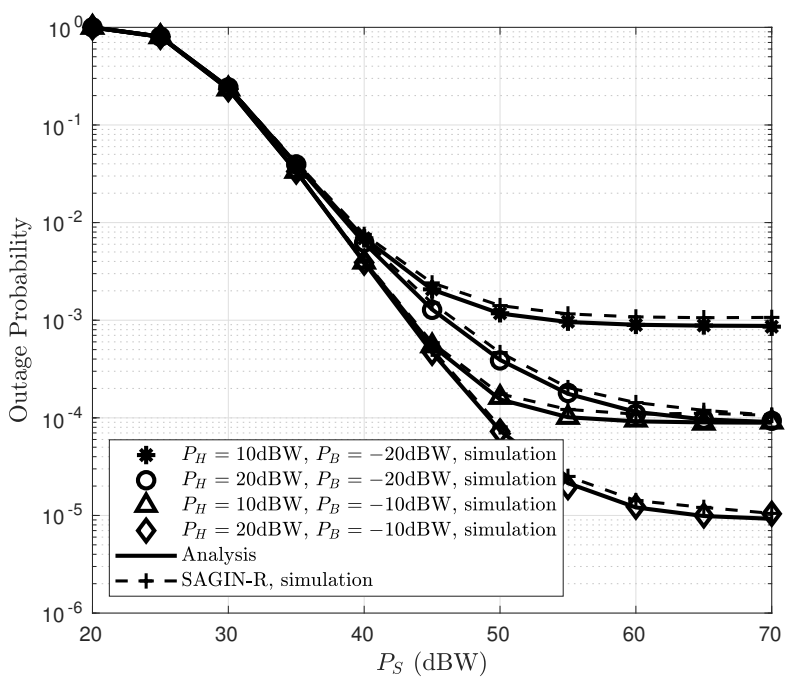

Fig. 6: System outage probability versus $P_{S}$.

$(8371,8371,9371000), \quad\left(x_{H}, y_{H}, z_{H}\right)=(178,145,20000)$, $\left(x_{B}, y_{B}, z_{B}\right)=(100,21,0),\left(x_{U}, y_{U}, z_{U}\right)=(223,293,0)$.

First, we show the impacts of the transmit power at GEO, HAP, and BS in Fig. 6. This figure illustrates the curves of the overall system outage probability for various $P_{H}$ and $P_{B}$. It is easy to observe that outage probability can be improved when the transmit power increases. However, an outage probability floor exists for each link in the high power regime, since the outage probabilities of HAP, BS, and UE links dominant the outage probability of the SAGIN system only when $\Phi_{S H}$ and $\Phi_{S B}$ are small. Moreover, under the identical ShadowedRician fading, the outage performance of the HAP-UE link and BS-UE link introduces a similar influence on the overall outage probability, when the HAP-BS link does not bring an apparent performance gain. In practice, user mobility is another critical issue which more or less affects system performance [47]. We also compare the system performance of the SAGIN system considering UE's mobility similar to that of HAP within radius $r_{U}=200 \mathrm{~m}$. This scenario is labeled as SAGIN-R in Fig. 6. Observing the simulation results compared to the scenarios without considering UE's mobility, we can find that the displacement of UE with a small radius does not greatly impact the outage performance because the radio propagation distance is much larger than the displacement of UE.

As shown in Fig. 7, the impact of $P_{S}$ on the five outage cases is observed. We can see that $\Phi_{1}$ increases as $P_{S}$ increases until it reaches a floor. The reason is that a higher transmit power at GEO brings a higher probability that HAP and BS could decode information correctly, but it is bounded by the outage probability of the HAP-UE and BS-UE links. $\Phi_{2}-\Phi_{3}$ experience increasing but then decreasing because all three cases consider one of GEO-UE or GEO-BS links is failed while the other one is successful. When the transmit power is small, the probability of successful transmission plays the main role since the outage probability is close to 1 . Conversely, the probability of failed transmission plays the main role as the

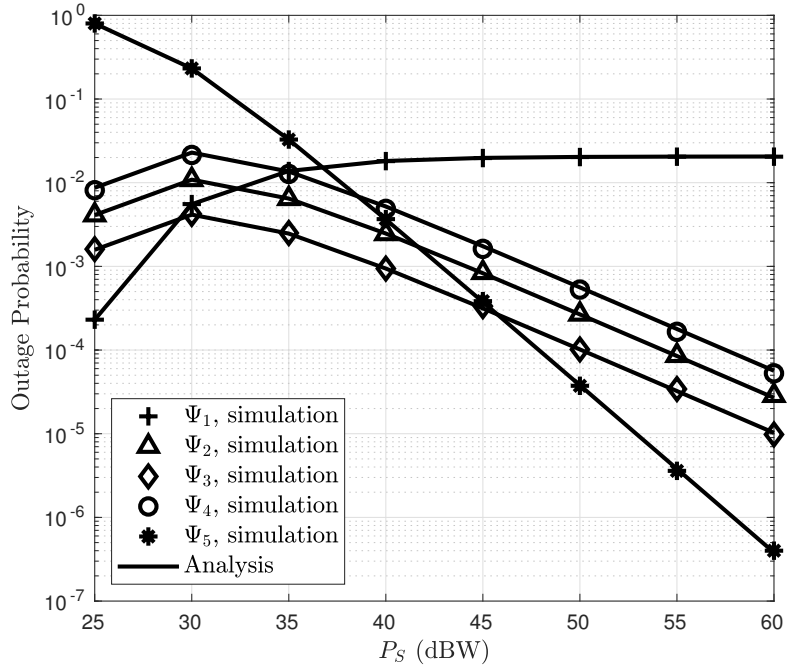

Fig. 7: Outage probability for each link versus $P_{S}$ with $P_{H}=-5$ $\mathrm{dBW} P_{B}=-30 \mathrm{dBW}$.

transmit power is high enough. Since $\Phi_{S B}$ and $\Phi_{S H}$ decrease as $P_{S}$ increases, $\Phi_{5}=\Phi_{S B} \Phi_{S H}$ also decreases.

In Fig. 8, we plot outage probability versus $r_{H}$ with various path loss exponents of the HAP-BS and HAP-UE links when $\left(x_{H}, y_{H}, z_{H}\right)=(178,145,20000)$. We can see that outage probability decreases as $r_{H}$ decreases and reaches the minimum value when $r_{H}$ reaches the smallest value under the condition that the path loss and the distance between HAPBS and HAP-UE links are moderate. Meanwhile, by changing the HAP position to $\left(x_{H}, y_{H}, z_{H}\right)=(5780,145,100)$ in Fig. 9, the minimal outage probability does not occur when $r_{H}$ takes the smallest value. It should be noted that the obtained solution to the formulated optimization problem is not the optimum, and the optimum may not be the smallest. However, since the height of HAP is around 20-25 kilometers in practical SAGIN systems, a small variation of $r_{H}$ will not make much difference in the distance between HAP and BS/UE so as the system outage performance. Therefore, the obtained outage performance can be regarded as near-optimal, and the difference between the optimal outage probability and suboptimal outage probability is trivial. It can also be explained by the fact that the distance between HAP and BS, as well as the distance between HAP and UE, become longer with a higher probability when HAP is allowed to move within a broader area. Moreover, outage probability achieves an upper bound as $r_{H}$ increases since $\Psi_{H B}$ and $\Psi_{H U}$ are close to 1 , and outage probability mainly depends on the performance of the remaining link. It is apparent that the path loss exponents have dominant impacts on the outage performance of the SAGIN system.

As depicted in Fig. 10, the asymptotic results for the outage probability obtained in Section III-D can accurately match the simulation and analysis in the high SNR regime. Compared to the results shown in Fig. 6, the first and the second transmission slots play different roles as the transmit power at GEO changes. The second transmission decides the outage 


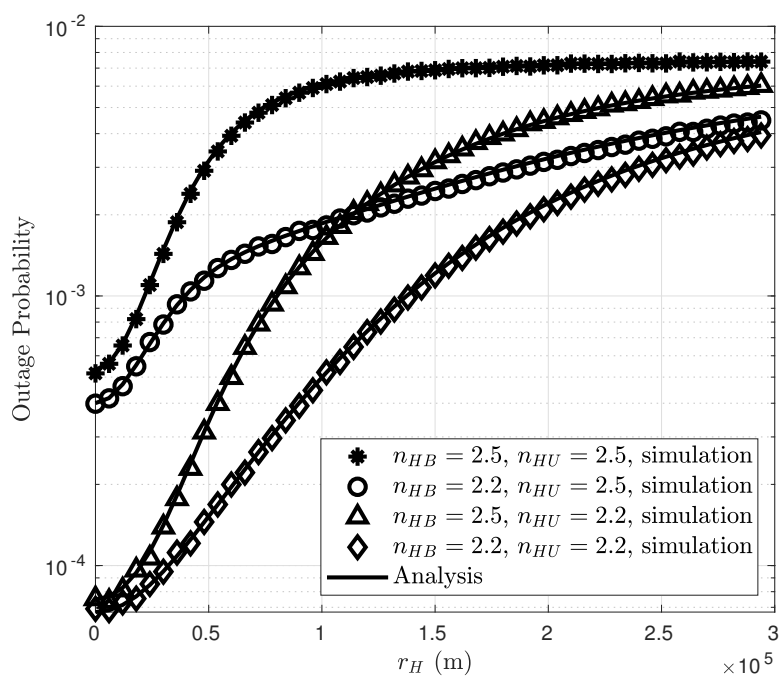

Fig. 8: System outage probability versus $r_{H}$ with $P_{S}=50 \mathrm{dBW}$, $P_{H}=20 \mathrm{dBW}$ and $P_{B}=-15 \mathrm{dBW}$.

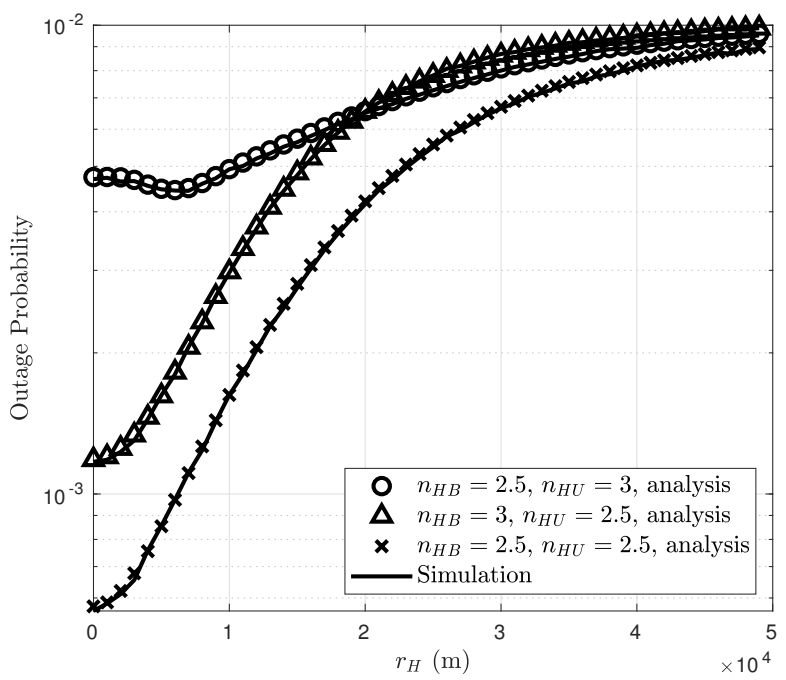

Fig. 9: System outage probability versus $r_{H}$ with $P_{S}=50 \mathrm{dBW}$, $P_{H}=10 \mathrm{dBW}$ and $P_{B}=-20 \mathrm{dBW}$.

floor when the transmission over the GEO-HAP and GEO-BS links achieve satisfying performance in the high power regime. The better the transmission in the first slot is, the higher the slope in the low power regime will be.

In addition, the direct link between GEO and UE might exist and bring a further performance gain [48], [49]. To be comprehensive, the performance compared to the SAGIN system with a direct link between GEO and UE (SAGIN-D) is shown in Fig. 11. We can see from this figure that there is a performance gap in the high $P_{S}$ regime because the outage performance of the GEO-UE link gets better as $P_{S}$ increases. Theoretically, the outage probability of SAGIN-D can be calculated by the outage probability of the direct link multiplying the derived outage probability of the SAGIN system without considering the direct link, that is, $\operatorname{Pr}\left\{\gamma_{S U}<\epsilon_{U}\right\} \Upsilon$. Therefore, when the signals are propagating over the direct link experience

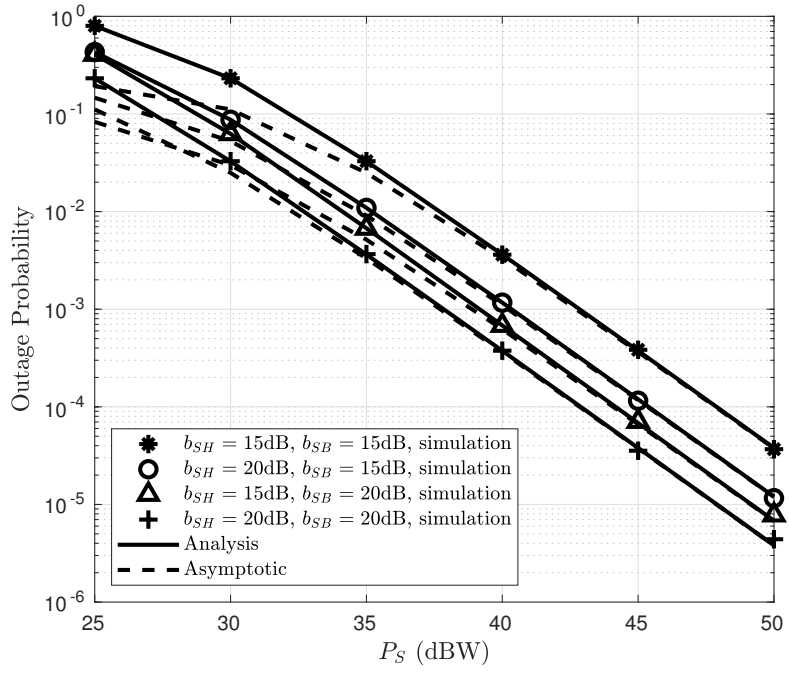

Fig. 10: System outage probability versus $P_{S}$ with $P_{H}=10 \mathrm{dBW}$ $+P_{S}$ and $P_{B}=-30 \mathrm{dBW}+P_{S}$.

severe attenuation, SAGIN's outage performance is close to that of SAGIN-D. In other words, our derived expression can be treated as a lower bound on the outage probability for the SAGIN-D system.

In Fig. 11, we also demonstrate the effects of channel estimation errors. Although we assume that the CSI is perfectly known for performance analysis, the CSI mismatch caused by channel estimation errors in practice will worsen the system performance [50]. Hence, the performance of the proposed system under imperfect CSI should be probed into. Assuming UE uses the outdated CSI to carry out the SC scheme, the outdated channel gain can be expressed as $\tilde{h}_{j}=\sqrt{\epsilon} h_{j}+\sqrt{1-\epsilon} \omega_{j}$, where $j \in\{H U, B U\} ; \epsilon$ is the correlation coefficient between $\tilde{h}_{j}$ and $h_{j} ; \omega_{j}$ is a random variable with the same distribution as $h_{j}$. Observing the results corresponding to different values of $\epsilon$, We can find that outdated/imperfect CSI greatly weakens the system outage performance and can even result in an outage floor in the high power regime.

The achievable data rate of the SAGIN system is shown in Fig. 12. It should be noted that the data rate of the SAGIN system should be discussed and simulated by two parallel cases:

1) The link between GEO and BS is available with probability $\operatorname{Pr}\left\{\gamma_{S B}>\epsilon_{B}\right\}$ with the end-to-end SNR $\gamma_{\text {end }}^{1}=$ $\max \left\{\min \left\{\gamma_{S B}, \gamma_{B U}\right\}, \min \left\{\gamma_{S H}, \gamma_{H U}\right\}\right\}$ at the user;

2) The link between GEO and BS is not available with probability $\operatorname{Pr}\left\{\gamma_{S B}<\epsilon_{B}\right\}$ with the end-to-end SNR $\gamma_{\text {end }}^{2}=\max \left\{\min \left\{\gamma_{H B}, \gamma_{B U}\right\}, \min \left\{\gamma_{S H}, \gamma_{H U}\right\}\right\}$ at the user.

It is clear from Fig. 12 that as $P_{S}$ increases, the achievable data rate decreases first and converge towards the same value for different $\epsilon_{B}$. This non-monotone trend is caused by the different dominant factors over the entire power regime. Specifically, the data rate is dominated by $\gamma_{H B}$ and $\gamma_{B U}$ of the second case in the low $P_{S}$ regime, while it is dominated by $\gamma_{B U}, \gamma_{S H}, \gamma_{H U}$ 


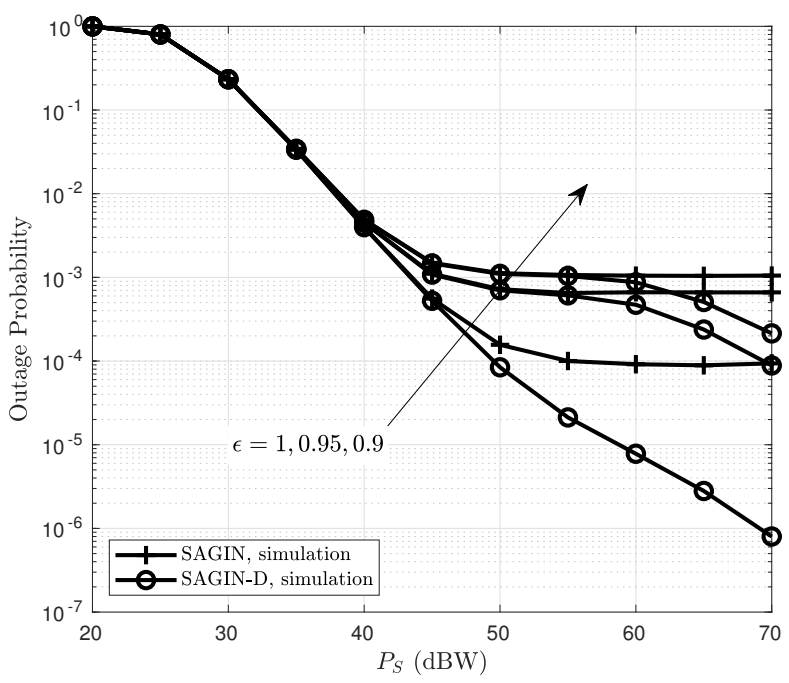

Fig. 11: System outage probability versus $P_{S}$ with $b_{S U}=\Omega_{S U}=15$ $\mathrm{dB}, m_{S U}=2.3, P_{H}=10 \mathrm{dBW} P_{B}=-20 \mathrm{dBW}$.

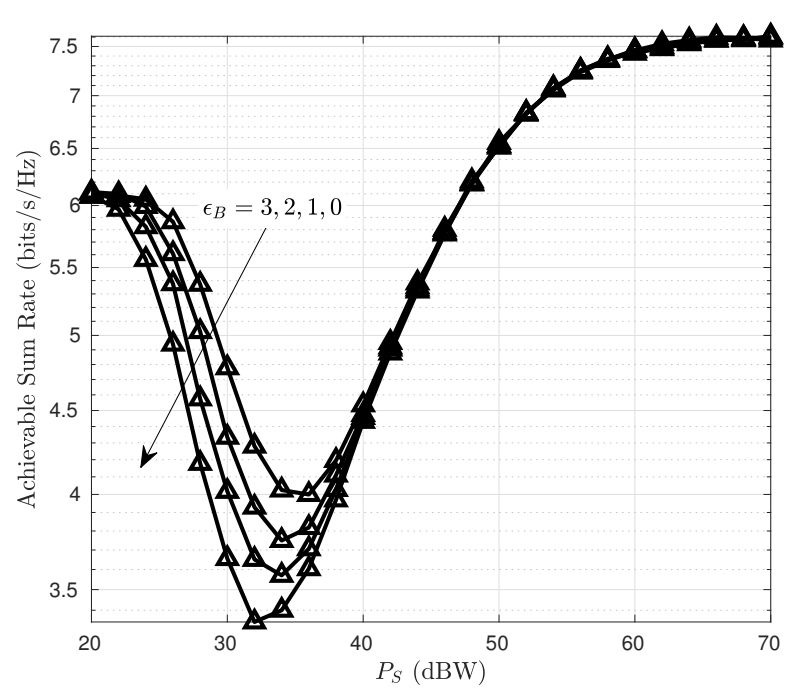

Fig. 12: Achievable data rate versus $P_{S}$ with $P_{H}=10 \mathrm{dBW}$ and $P_{B}=-20 \mathrm{dBW}$.

in the high $P_{S}$ regime. Besides, we can also observe that a higher SNR threshold at BS can improve the data rate in the low $P_{S}$ regime, since it increases the probability of the second case that is more reliable and can provide a higher data rate.

Moreover, we make a comparison with a standard benchmark system called the GEO-BS-UE (GBU) system, where there are only GEO-BS and BS-UE links without the assistant of HAP. For fair comparison in performance, we assume GEO, $\mathrm{BS}$, and UE located in the same position, and GEO-BS and BS-UE links experience the same fading as in our proposed SAGIN system. As can be seen in Fig. 13, SAGIN greatly outperforms GBU in terms of outage performance with the additional $1 \mathrm{dBW}$ transmit power at HAP. With the same $P_{B}$, the GEO in GBU needs about $8 \mathrm{dBW}$ extra power to achieve the same 0.1 outage probability as SAGIN. It shows that GBU consumes more power to gain better outage performance,

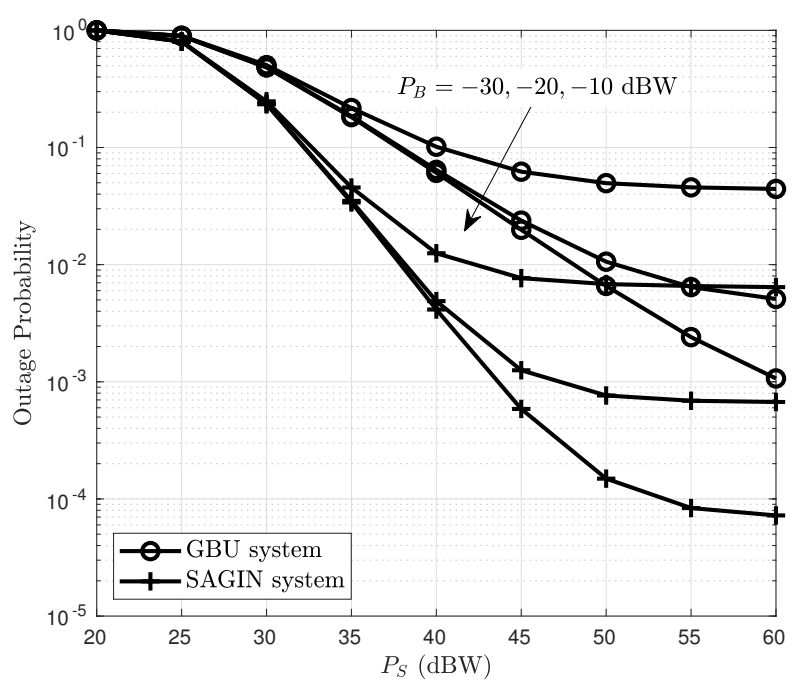

Fig. 13: System outage probability versus $P_{S}$ with $P_{H}=1 \mathrm{dBW}$.

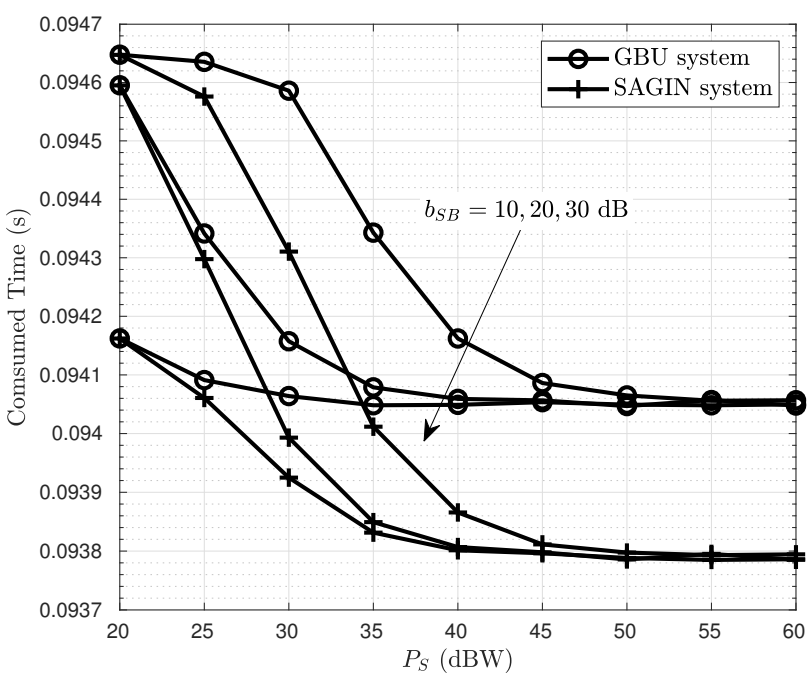

Fig. 14: Consumed time of 1 bit information transmission versus $P_{S}$ with $P_{H}=10 \mathrm{dBW}$.

which also verifies the superiority of our proposed SAGIN system.

In the final experiment, we compare the transmission time for 1 bit information of our proposed SAGIN network with the GBU network in Fig. 14. With additional HAP-BS and HAP-UE links, the SAGIN system consumes much less time than the GBU system. The consumed time of both systems approaches a floor as $P_{S}$ increases. However, the achieved floor of the SAGIN system is much lower than the one of the GBU system because of the improved performance brought by the HAP. Although the GBU system could transmit the same bit information within a shorter time with better channel condition than the SAGIN system in the low $P_{S}$ regime, it will be surpassed by the SAGIN system in the high $P_{S}$ regime. It also shows that the GBU system needs about 5 $\mathrm{dBW}$ or more transmit power at the satellite to achieve the 
same transmission time consumed by the SAGIN system. This is highly undesirable because satellites have limited sources of transmission energy, and most of them might not be rechargeable.

\section{CONCLUSION}

In this work, we analyzed a SAGIN system from the cooperative perspective, where the cooperating HAP and BS assist the transmission from GEO to UE. All outage scenarios were presented with classified space channels, aerial channels, and a terrestrial channel. Taking the HAP mobility into consideration, we analyzed the system outage performance from the perspective of each link. The approximated and asymptotic closed-form expressions for outage probabilities of each link have been derived as well as the outage probability of the SAGIN system. After that, we studied the outage performance optimization problem by finding the maximum placement distance of the HAP. Due to the mathematical intractability of the original objective function, we proposed an alternative optimization problem, which is capable of providing suboptimal solutions with low complexity. The correctness of the outage performance analysis has been verified through Monte Carlo simulations. Through a fair comparison with the benchmark GEO-BS-UE system regarding outage performance and transmission time, we revealed the superiority of our proposed SAGIN system.

\section{ACKNOWLEDGMENT}

The authors would like to thank the editor and the anonymous reviewers for their constructive comments which help improve the quality of this paper.

\section{REFERENCES}

[1] S. Dang, O. Amin, B. Shihada, and M.-S. Alouini, "What should 6G be?" Nature Electronics., vol. 3, no. 1, pp. 20-29, Jan. 2020.

[2] M. Dohler, R. W. Heath, A. Lozano, C. B. Papadias, and R. A. Valenzuela, "Is the PHY layer dead?" IEEE Communications Magazine, vol. 49, no. 4, pp. 159-165, Apr. 2011.

[3] J. Liu, Y. Shi, Z. M. Fadlullah, and N. Kato, "Space-air-ground integrated network: A survey," IEEE Communications Surveys Tutorials, vol. 20, no. 4, pp. 2714-2741, Fourthquarter 2018.

[4] P. Chini, G. Giambene, and S. Kota, "A survey on mobile satellite systems," International Journal of Satellite Communications and Networking, vol. 28, no. 1, pp. 29-57, 2010.

[5] M. Giordani and M. Zorzi, "Non-terrestrial communication in the 6G era: Challenges and opportunities," arXiv preprint arXiv:1912.10226, 2019.

[6] B. Li, Z. Fei, and Y. Zhang, "Uav communications for $5 \mathrm{G}$ and beyond: Recent advances and future trends," IEEE Internet of Things Journal, vol. 6, no. 2, pp. 2241-2263, Apr. 2019.

[7] V. P. Hubenko, R. A. Raines, R. F. Mills, R. O. Baldwin, B. E. Mullins, and M. R. Grimaila, "Improving the Global Information Grid's performance through satellite communications layer enhancements," IEEE Communications Magazine, vol. 44, no. 11, pp. 66-72, Nov. 2006.

[8] G. Xiong, F. Zhu, X. Dong, H. Fan, B. Hu, Q. Kong, W. Kang, and T. Teng, "A kind of novel ITS based on space-air-ground big-data," IEEE Intelligent Transportation Systems Magazine, vol. 8, no. 1, pp. 10-22, Spring 2016.

[9] Y. Wang, Y. Xu, Y. Zhang, and P. Zhang, "Hybrid satellite-aerialterrestrial networks in emergency scenarios: a survey," China Соттиnications, vol. 14, no. 7, pp. 1-13, Jul. 2017.

[10] E. Yaacoub and M.-S. Alouini, "A key 6G challenge and opportunityconnecting the base of the pyramid: A survey on rural connectivity," 2020.
[11] N. Zhang, S. Zhang, P. Yang, O. Alhussein, W. Zhuang, and X. S. Shen, "Software defined space-air-ground integrated vehicular networks: Challenges and solutions," IEEE Communications Magazine, vol. 55, no. 7, pp. 101-109, Jul. 2017.

[12] Z. Zhou, J. Feng, C. Zhang, Z. Chang, Y. Zhang, and K. M. S. Huq, "SAGECELL: Software-defined space-air-ground integrated moving cells," IEEE Communications Magazine, vol. 56, no. 8, pp. 92-99, Aug. 2018.

[13] Y. Shi, Y. Cao, J. Liu, and N. Kato, "A cross-domain SDN architecture for multi-layered space-terrestrial integrated networks," IEEE Network, vol. 33, no. 1, pp. 29-35, Jan. 2019.

[14] Y. Shi, J. Liu, Z. M. Fadlullah, and N. Kato, "Cross-layer data delivery in satellite-aerial-terrestrial communication," IEEE Wireless Communications, vol. 25, no. 3, pp. 138-143, Jun. 2018.

[15] S. Zhou, G. Wang, S. Zhang, Z. Niu, and X. S. Shen, "Bidirectional mission offloading for agile space-air-ground integrated networks," IEEE Wireless Communications, vol. 26, no. 2, pp. 38-45, Apr. 2019.

[16] N. Kato, Z. Md. Fadlullah, F. Tang, B. Mao, S. Tani, A. Okamura, and J. Liu, "Optimizing space-air-ground integrated networks by artificial intelligence," IEEE Wireless Communications, pp. 1-8, 2019.

[17] I. A. Alimi, A. O. Mufutau, A. L. Teixeira, and P. P. Monteiro, "Performance analysis of space-air-ground integrated network (SAGIN) over an arbitrarily correlated multivariate FSO channel," Wireless Personal Communications, vol. 100, no. 1, pp. 47-66, 2018.

[18] I. A. Alimi, A. L. Teixeira, and P. P. Monteiro, "Effects of correlated multivariate FSO channel on outage performance of space-air-ground integrated network (SAGIN)," Wireless Personal Communications, pp. 1-19, 2019.

[19] Y. Ruan, Y. Li, C. Wang, and R. Zhang, "Energy efficient adaptive transmissions in integrated satellite-terrestrial networks with SER constraints," IEEE Transactions on Wireless Communications, vol. 17, no. 1, pp. 210-222, Jan. 2018.

[20] J. N. Pelton, S. Madry, and S. Camacho-Lara, Handbook of satellite applications. Springer New York, 2017.

[21] X. Yan, H. Xiao, C.-X. Wang, and K. An, "Outage performance of NOMA-based hybrid satellite-terrestrial relay networks," IEEE Wireless Communications Letters, vol. 7, no. 4, pp. 538-541, 2018.

[22] K. F. Hasan, Y. Feng, and Y. Tian, "Gnss time synchronization in vehicular ad-hoc networks: Benefits and feasibility," IEEE Transactions on Intelligent Transportation Systems, vol. 19, no. 12, pp. 3915-3924, 2018.

[23] H. Li, L. Han, R. Duan, and G. M. Garner, "Analysis of the synchronization requirements of $5 \mathrm{~g}$ and corresponding solutions," IEEE Communications Standards Magazine, vol. 1, no. 1, pp. 52-58, 2017.

[24] W. Wang, Y. Tong, L. Li, A.-A. Lu, L. You, and X. Gao, "Near optimal timing and frequency offset estimation for $5 \mathrm{~g}$ integrated leo satellite communication system," IEEE Access, vol. 7, pp. 113298-113310, 2019.

[25] M. Arti, "Channel estimation and detection in hybrid satellite-terrestrial communication systems," IEEE Transactions on Vehicular Technology, vol. 65 , no. 7 , pp. 5764-5771, 2015.

[26] _ - "Channel estimation and detection in satellite communication systems," IEEE Transactions on Vehicular Technology, vol. 65, no. 12, pp. $10173-10179,2016$.

[27] R. Xu, X. Da, H. Hu, L. Ni, and Y. Pan, "Self-interference cancellation scheme for secure af satellite communication based on fh-mwfrft," IEEE Communications Letters, vol. 23, no. 11, pp. 2050-2053, 2019.

[28] H. Ghannam and I. Darwazeh, "Sefdm over satellite systems with advanced interference cancellation," IET Communications, vol. 12, no. 1, pp. 59-66, 2017.

[29] M. Haenggi, "On distances in uniformly random networks," IEEE Transactions on Information Theory, vol. 51, no. 10, pp. 3584-3586, Oct. 2005.

[30] A. Behnad and N. C. Beaulieu, "Best neighbor communication in a Poisson field of nodes," IEEE Transactions on Vehicular Technology, vol. 64, no. 2, pp. 818-823, Feb. 2015.

[31] I. M. Kostic, "Analytical approach to performance analysis for channel subject to shadowing and fading," IEE Proceedings - Communications, vol. 152, no. 6, pp. 821-827, Dec. 2005.

[32] W. Yi, Y. Liu, Y. Deng, and A. Nallanathan, "Clustered uav networks with millimeter wave communications: A stochastic geometry view," IEEE Transactions on Communications, pp. 1-1, 2020.

[33] T. Z. H. Ernest, A. S. Madhukumar, R. P. Sirigina, and A. K. Krishna, "NOMA-aided UAV communications over correlated rician shadowed fading channels," IEEE Transactions on Signal Processing, vol. 68, pp. 3103-3116, 2020 
[34] C. Loo, "A statistical model for a land mobile satellite link," IEEE Transactions on Vehicular Technology, vol. 34, no. 3, pp. 122-127, Aug. 1985.

[35] A. Abdi, W. C. Lau, M.-S. Alouini, and M. Kaveh, "A new simple model for land mobile satellite channels: first- and second-order statistics," IEEE Transactions on Wireless Communications, vol. 2, no. 3, pp. 519528, May 2003.

[36] I. S. Gradshteyn and I. M. Ryzhik, Table of Integrals, Series, and Products. Academic Press, 2014.

[37] T. Pelzmann, T. Jost, M. Schwinzerl, F. Perez-Fontán, M. Schonhuber, and N. Floury, "Airborne measurements enhancing the satellite-toaircraft channel model in L-band," in Proc. IEEE EuCAP, Davos, Switzerland, Apr. 2016, pp. 1-5.

[38] Y. Zeng, R. Zhang, and T. J. Lim, "Wireless communications with unmanned aerial vehicles: Opportunities and challenges," IEEE Communications Magazine, vol. 54, no. 5, pp. 36-42, 2016.

[39] J. Proakis and M. Salehi, Digital Communications. McGraw-Hill, 2008.

[40] S. Dang, J. P. Coon, and G. Chen, "Outage performance of two-hop OFDM systems with spatially random decode-and-forward relays," IEEE Access, vol. 5, pp. 27 514-27 524, 2017.

[41] K. An, M. Lin, J. Ouyang, and W. Zhu, "Secure transmission in cognitive satellite terrestrial networks," IEEE Journal on Selected Areas in Communications, vol. 34, no. 11, pp. 3025-3037, Nov. 2016.

[42] M. Z. Bocus, C. P. Dettmann, and J. P. Coon, "An approximation of the first order marcum Q-function with application to network connectivity analysis," IEEE Communications Letters, vol. 17, no. 3, pp. 499-502, Mar. 2013.

[43] M. K. Arti and V. Jain, "Relay selection-based hybrid satellite-terrestrial communication systems," IET Communications, vol. 11, no. 17, pp. 2566-2574, 2017.

[44] X. Yan, H. Xiao, K. An, G. Zheng, and W. Tao, "Hybrid satellite terrestrial relay networks with cooperative non-orthogonal multiple access," IEEE Communications Letters, vol. 22, no. 5, pp. 978-981, 2018.

[45] K. An, M. Lin, T. Liang, J. Wang, J. Wang, Y. Huang, and A. L. Swindlehurst, "Performance analysis of multi-antenna hybrid satellite-terrestrial relay networks in the presence of interference," IEEE Transactions on Communications, vol. 63, no. 11, pp. 4390-4404, 2015.

[46] P. K. Sharma, P. K. Upadhyay, D. B. da Costa, P. S. Bithas, and A. G. Kanatas, "Performance analysis of overlay spectrum sharing in hybrid satellite-terrestrial systems with secondary network selection," IEEE Transactions on Wireless Communications, vol. 16, no. 10, pp. 6586-6601, 2017.

[47] X. Yan, H. Xiao, K. An, G. Zheng, and S. Chatzinotas, "Ergodic capacity of NOMA-based uplink satellite networks with randomly deployed users," IEEE Systems Journal, pp. 1-8, 2019.

[48] K. An, Y. Li, X. Yan, and T. Liang, "On the performance of cacheenabled hybrid satellite-terrestrial relay networks," IEEE Wireless Communications Letters, vol. 8, no. 5, pp. 1506-1509, 2019.

[49] K. An and T. Liang, "Hybrid satellite-terrestrial relay networks with adaptive transmission," IEEE Transactions on Vehicular Technology, vol. 68, no. 12, pp. 12448-12 452, 2019.

[50] W. Lu, K. An, and T. Liang, "Robust beamforming design for sum secrecy rate maximization in multibeam satellite systems," IEEE Transactions on Aerospace and Electronic Systems, vol. 55, no. 3, pp. 15681572, 2019. 